\title{
Lyman continuum escape fraction of faint galaxies at $z \sim 3.3$ in the CANDELS/GOODS-North, EGS, and COSMOS fields with LBC ${ }^{\star}$
}

\author{
A. Grazian ${ }^{1}$, E. Giallongo ${ }^{1}$, D. Paris ${ }^{1}$, K. Boutsia ${ }^{2}$, M. Dickinson ${ }^{3}$, P. Santini ${ }^{1}$, R. A. Windhorst ${ }^{4}$, R. A. Jansen ${ }^{4}$,
} S. H. Cohen ${ }^{4}$, T. A. Ashcraft ${ }^{4}$, C. Scarlata ${ }^{5}$, M. J. Rutkowski ${ }^{6}$, E. Vanzella ${ }^{7}$, F. Cusano ${ }^{7}$, S. Cristiani ${ }^{8}$, M. Giavalisco ${ }^{9}$, H. C. Ferguson ${ }^{10}$, A. Koekemoer ${ }^{10}$, N. A. Grogin ${ }^{10}$, M. Castellano ${ }^{1}$, F. Fiore ${ }^{1}$, A. Fontana ${ }^{1}$, F. Marchi ${ }^{1}$, F. Pedichini ${ }^{1}$, L. Pentericci ${ }^{1}$, R. Amorín ${ }^{11,12}$, G. Barro ${ }^{13}$, A. Bonchi ${ }^{14,1}$, A. Bongiorno ${ }^{1}$, S. M. Faber ${ }^{15}$, M. Fumana ${ }^{16}$, A. Galametz ${ }^{17}$, L. Guaita ${ }^{18,1}$, D. D. Kocevski ${ }^{19}$, E. Merlin ${ }^{1}$, M. Nonino ${ }^{8}$, R. W. O’Connell ${ }^{20}$, S. Pilo ${ }^{1}$, R. E. Ryan ${ }^{10}$, E. Sani ${ }^{18}$, R. Speziali ${ }^{1}$, V. Testa ${ }^{1}$, B. Weiner ${ }^{21}$, and H. Yan ${ }^{22}$

(Affiliations can be found after the references)

Received 16 January 2017 / Accepted 28 February 2017

\begin{abstract}
Context. The reionization of the Universe is one of the most important topics of present-day astrophysical research. The most plausible candidates for the reionization process are star-forming galaxies, which according to the predictions of the majority of the theoretical and semi-analytical models should dominate the $\mathrm{H}$ I ionizing background at $z \gtrsim 3$.

Aims. We measure the Lyman continuum escape fraction, which is one of the key parameters used to compute the contribution of star-forming galaxies to the UV background. It provides the ratio between the photons produced at $\lambda \leq 912 \AA$ rest-frame and those that are able to reach the inter-galactic medium, i.e. that are not absorbed by the neutral hydrogen or by the dust of the galaxy's inter-stellar medium.

Methods. We used ultra-deep $U$-band imaging $(U=30.2$ mag at $1 \sigma)$ from Large Binocular Camera at the Large Binocular Telescope (LBC/LBT) in the CANDELS/GOODS-North field and deep imaging in the COSMOS and EGS fields in order to estimate the Lyman continuum escape fraction of 69 star-forming galaxies with secure spectroscopic redshifts at $3.27 \leq z \leq 3.40$ to faint magnitude limits $\left(L=0.2 L^{*}\right.$, or equivalently $\left.M_{1500} \sim-19\right)$. The narrow redshift range implies that the LBC $U$-band filter exclusively samples the $\lambda \leq 912 \AA$ rest-frame wavelengths.

Results. We measured through stacks a stringent upper limit $(<1.7 \%$ at $1 \sigma)$ for the relative escape fraction of $\mathrm{HI}$ ionizing photons from bright galaxies $\left(L>L^{*}\right)$, while for the faint population $\left(L=0.2 L^{*}\right)$ the limit to the escape fraction is $\lesssim 10 \%$. We computed the contribution of star-forming galaxies to the observed UV background at $z \sim 3$ and find that it is not sufficient to keep the Universe ionized at these redshifts unless their escape fraction increases significantly $(\geq 10 \%)$ at low luminosities $\left(M_{1500} \geq-19\right)$.

Conclusions. We compare our results on the Lyman continuum escape fraction of high- $z$ galaxies with recent estimates in the literature, and discuss future prospects to shed light on the end of the Dark Ages. In the future, strong gravitational lensing will be fundamental in order to measure the Lyman continuum escape fraction down to faint magnitudes $\left(M_{1500} \sim-16\right)$ that are inaccessible with the present instrumentation on blank fields. These results will be important in order to quantify the role of faint galaxies to the reionization budget.
\end{abstract}

Key words. galaxies: distances and redshifts - galaxies: evolution - galaxies: high-redshift - galaxies: photometry

\section{Introduction}

The reionization of the Universe, related to the end of the socalled Dark Ages, is now located in the redshift interval $z=$ 6.0-8.8. The lower limit is derived from observations of the Gunn-Peterson effect in luminous $z>6$ quasar (QSO) spectra (Fan et al. 2006), while the most recent upper limit, $z<$ 8.8, comes from measurements of the Thomson optical depth $\tau_{\mathrm{e}}=0.055 \pm 0.009$ in the CMB polarization map by Planck (Planck Collaboration XLVII 2016). These limits are consistent with a rapid $\mathrm{H}$ I reionization at $z_{\text {reion }}=7.8 \pm 1.0$.

These main results have been corroborated by a number of confirmations: the kinetic Sunyaev-Zel'dovich (kSZ) effect measured by the South Pole Telescope (SPT, Zahn et al. 2012), when combined with the recent Planck Collaboration XLVII (2016) maps gives a stringent limit of $\Delta z_{\text {reion }}<2.8$ to the duration of reionization. Moreover, the redshift evolution of the

\footnotetext{
* Based on observations made at the Large Binocular Telescope (LBT) at Mt. Graham (Arizona, USA).
}

luminosity function (Konno et al. 2014) of Lyman- $\alpha$ emitters (LAEs) and the sudden drop observed at $z \sim 7$ in the fraction of Lyman-break galaxies (LBGs) with strong Lyman- $\alpha$ in emission (e.g., Fontana et al. 2010; Pentericci et al. 2011, 2014; Ono et al. 2012; Treu et al. 2012; Schenker et al. 2014) are indicating that the Universe is becoming more neutral at $z>7$. All these observations favor a late and possibly inhomogeneous reionization process.

While we now have significant information regarding the timing of the reionization process, we are still looking for the sources that provide the bulk of the $\mathrm{HI}$ ionizing photons. To date, obvious candidates have been searched for among highredshift star-forming galaxies (SFGs) and/or active galactic nuclei (AGNs).

At high redshift, bright QSOs $\left(M_{1450} \leq-27\right)$ are quite rare as their space density declines rapidly. Their ionizing emissivity is quite low, and there are not enough of them to keep the intergalactic medium (IGM) ionized at $z>3$ (Haardt \& Madau 1996; Cowie et al. 2009). However Glikman et al. (2011) and 
Giallongo et al. (2015) have recently found a significant population of fainter AGNs $\left(-25 \leq M_{1450} \leq-19\right)$ at $z \geq 4$, which could possibly provide the whole ionizing emissivity at high- $z$ (Madau \& Haardt 2015; Khaire et al. 2016).

In the last 15 years much effort has been given to high- $z$ starforming galaxies as emitters of ionizing radiation. This choice has been driven by four considerations: 1) early WMAP results, heavily affected by dust polarization of the Milky Way, indicated a much earlier reionization epoch, around $z \geq 12$ (Hinshaw et al. 2013); 2) the luminosity function of galaxies is gradually steepening with redshifts at $z \geq 4$ (Finkelstein et al. 2015; Bouwens et al. 2015); 3) numerous galaxies exist even at very faint absolute magnitudes $\left(M_{1500} \sim-12\right)$ at $z \geq 6$ (Livermore et al. 2017); and 4) at high redshifts galaxies could be efficient producers of ionizing photons (Bouwens et al. 2016b), whose possible contribution crucially depends on their ability to carve ionizing bubbles in their neighborhood, which is quantified by the Lyman Continuum (LyC) escape fraction parameter.

At $z \sim 3-4.5$, the highest redshift where the IGM absorption does not prevent this kind of measure, we still do not have a reliable estimate of the average escape fraction from the star-forming galaxy population. A few claimed detections (Steidel et al. 2001; Shapley et al. 2006; Nestor et al. 2011; Mostardi et al. 2013) have been called into question, due to possible contamination by low redshift interlopers (e.g., Vanzella et al. 2012a). Other datasets provide marginal detections (Marchi et al. 2017) or upper limits at levels of only a few percent (Giallongo et al. 2002; Grimes et al. 2009; Cowie et al. 2009; Siana et al. 2010; Bridge et al. 2010; Vanzella et al. 2010; Boutsia et al. 2011; Leitet et al. 2013; Grazian et al. 2016; Guaita et al. 2016; Japelj et al. 2017).

Very recently the situation has improved, especially at very low redshifts, thanks to observations by the COS instrument on board HST. Escape fractions within 6-13\% have been detected in five galaxies with high [OIII]/[OII] line ratios and high ionizing photon production efficiency. These ionizers appear compact with high star formation rate surface density $\Sigma_{\mathrm{SFR}}$ (Izotov et al. 2016a,b; Verhamme et al. 2017; Schaerer et al. 2016), resembling properties typical of Green Pea galaxies (Cardamone et al. 2009; Amorin et al. 2010, 2012a; Henry et al. 2015). This key feature of the local ionizers induces a strong feedback on their ISM, through rapid outflows, which may be physically related to leakage of Lyman continuum radiation, as suggested by, e.g., Heckman et al. (2011), Amorin et al. (2012b), and Borthakur et al. (2014), and explored further with simulations by Sharma et al. (2016, 2017). High [OIII]/[OII] line ratios seen in low- $z$ blue compact dwarfs (BCDs) and high- $z$ galaxies may be attributed to many factors such as low metallicity, high ionization parameters, hard ionizing radiation fields, and/or the presence of density-bound H II regions (Stasinska et al. 2015; Steidel et al. 2016; Nakajima et al. 2016). Similar results were also found by Leitet et al. (2013), Bergvall et al. (2013), Borthakur et al. (2014), and Leitherer et al. (2016). Three out of the five galaxies described by Izotov et al. (2016b) were detected by the Widefield Infrared Survey Explorer (WISE) at 22 micron, possibly indicating the presence of warm/hot dust, heated by a buried starburst or by an AGN. The luminosities of these LyC emitters are between $M_{\mathrm{UV}}=-20.4$ and -21.3 , thus around $L^{*}(z=3)$, hereafter $L^{*}$. These five galaxies were selected from a sample of $\sim 10^{4}$ SDSS galaxies at $z \sim 0.3$, so it is not clear whether they represent a sparse minority of the SDSS sample, selected thanks to their peculiar properties (i.e., high [OIII]/[OII] line ratios).
Vanzella et al. (2016) and de Barros et al. (2016) found the first clear evidence of a LyC emitter at $z \sim 3.2$ free from interloper contamination. This galaxy, named Ion2, has peculiar properties similar to the five galaxies from Izotov et al. (2016b) mentioned above. Ion 2 has a line ratio of $[\mathrm{OIII}] /[\mathrm{OII}]>10$, strong Lyman- $\alpha$ in emission and compact morphology. Galaxies with similar properties have been recently discovered and characterized by Amorin et al. (2017), who showed that they are relatively rare and good analogs of primeval galaxies at $z>6$. However, the spectral and physical properties of these objects are rather peculiar, and it is not clear whether a significant population with similar properties is common at high redshifts (Faisst 2016; Khostovan et al. 2016). Recently, Naidu et al. (2016) have found six LyC candidates out of 1124 galaxies at $z \sim 2$ in the GOODS fields, confirming the peculiarity of these LyC emitters. Interestingly, at least $50 \%$ of these ionizing sources are confirmed as AGNs with large escape fraction $(\gtrsim 60 \%)$.

Given the null result on the brighter SFGs, a crucial contribution on the reionization process by a faint population $\left(L<L^{*}\right)$ at high redshift has been conjectured. This hypothesis has also been predicted by some theoretical models (e.g., Yajima et al. 2011; Razoumov \& Sommer-Larsen 2010; but see also models by Gnedin et al. 2008; Ma et al. 2015; Sharma et al. 2016, 2017, for opposite conclusions).

From an observational perspective, however, there are several pieces of evidence suggesting that low luminosity galaxies may not contribute appreciably to the H I ionizing background. Among the most interesting results, there are the large-scale opacity fluctuations observed in the Lyman- $\alpha$ forest of high- $z$ QSOs by Becker et al. (2015). The large variance in the spatial scales of the transmission of the IGM at $z \sim 5.5-6.0$ has been interpreted as being due to bright and rare sources rather than to a diffuse population of faint galaxies (e.g., Chardin et al. 2017). This indication has been strengthen by the non-detection of LyC flux (escape fraction $<2 \%$ at $3 \sigma$ ) from a large sample of moderately star-forming galaxies at $z \sim 1$ observed by Rutkowski et al. (2016).

Additional observational evidence is related to the sudden decrease in the number density of Lyman- $\alpha$ emitters at $z \gtrsim 6$. According to predictions by Dijkstra et al. (2016), the escape of Lyman- $\alpha$ photons should be connected with the leakage of LyC radiation, since they are possibly linked to clear sight lines in neutral hydrogen. The observed drop in Lyman- $\alpha$ is interpreted as being due to a rapid evolution of the ionization fraction of the intergalactic medium (IGM) in the Universe from $z=6$ to $z=7-8$ (Fontana et al. 2010; Pentericci et al. 2011, 2014; Schmidt et al. 2016). Moreover, the decrease in number density is differential, with a less pronounced and slower drop for brighter galaxies (Matthee et al. 2015; Santos et al. 2016; Vanzella et al. 2014; Oesch et al. 2015; Stark et al. 2017), which can be described as "downsizing reionization". This behavior is commonly interpreted as a signature of inhomogeneous and patchy reionization (Treu et al. 2012; Pentericci et al. 2014), where ionizing bubbles expand first in cosmic time around brighter and more massive galaxies, possibly associated with overdense regions (Castellano et al. 2016). The suggested average downsizing scenario for the luminosity of the ionizing sources would possibly imply a minor contribution, if any, of faint galaxies to the reionization process. To improve our knowledge of Lyman continuum emitters among the SFGs, we started to analyze a relatively large sample of galaxies at $z \sim 3$, paying specific attention to the fainter population.

In the COSMOS field, the LyC escape fraction of 45 bright $\left(L \geq 0.5 L^{*}\right)$ star-forming galaxies at $z \sim 3.3$ has been studied 
by Boutsia et al. (2011) and Grazian et al. (2016). In a companion paper by Guaita et al. (2016) we enlarged this sample by adding 86 bright galaxies in the Extended Chandra Deep Field South. In the present paper we explore the HI ionizing contribution of fainter galaxies in the CANDELS/GOODS-North and EGS fields with the addition of crucial and unique ultra-deep UV imaging from the LBC imager at the Large Binocular Telescope. We have also included in the analysis three galaxies affected by strong lensing, with the aim of probing the possible LyC contribution by intrinsically low luminosity galaxies down to $L \sim 0.04 L^{*}$.

This paper is organized as follows: in Sect. 2 we present the dataset; in Sect. 3 we describe the method adopted; in Sect. 4 we show the results for individual objects and for the overall sample as a whole; in Sect. 5 we provide an estimate of the ionizing background produced by these galaxies; in Sect. 6 we discuss our results; and in Sect. 7 we provide a summary and the conclusions. Throughout the paper we adopt the $\Lambda$-CDM concordance cosmological model $\left(H_{0}=70 \mathrm{~km} \mathrm{~s}^{-1} \mathrm{Mpc}^{-1}, \Omega_{\mathrm{M}}=0.3\right.$, and $\Omega_{\Lambda}=0.7$ ), consistent with recent CMB measurements (Planck Collaboration XLVII 2016). All magnitudes are in the $\mathrm{AB}$ system. All the limits to the escape fraction in this paper are at a $1 \sigma$ level, unless we state otherwise.

\section{Data}

The sample used in the present analysis is based on new ultradeep UV images obtained with the LBC instrument of spectroscopically confirmed galaxies in the GOODS-North field coupled with moderately deep LBC images in the EGS/AEGIS field and additional data in the COSMOS field, collected from the literature as described in the following.

\subsection{COSMOS, Q0933+28, and $Q 1623+26$ fields}

We started this project with deep LBC imaging of the COSMOS, Q0933+28, and Q1623+26 fields in the $U$ and $R$ bands, which have been used by Boutsia et al. (2011) and Grazian et al. (2016) to study the LyC escape fraction of 11 and 34 star-forming galaxies, respectively, with spectroscopic redshifts $\sim 3.3$ from the VIMOS Ultra-Deep Survey (VUDS, Le Fèvre et al. 2015). They constitute a mix of Lyman- $\alpha$ emitters and Lyman-Break galaxies. This starting sample of 45 galaxies was visually inspected against contamination by foreground objects and it has been discussed in detail in Grazian et al. (2016).

We added to this starting sample seven galaxies with spectroscopic redshifts $3.27<z<3.40$ from Onodera et al. (2016) in the same COSMOS area covered by deep LBC data in the $U$ and $R$ bands. All these galaxies have magnitudes between $R=24.5$ and 26.0, with the exception of a very faint source with $R \sim 27$, and all seven galaxies have a flux ratio between [OIII] and [OII] of $\sim 1.5-3$ and a SFR of $\sim 4-70 M_{\odot} / \mathrm{yr}$. An additional bright $(R=22.95)$ star-forming galaxy (ID = 53167 in Table 1$)$ with Lyman- $\alpha$ in emission at $z=3.359$ was found serendipitously during a spectroscopic campaign with the LBT MODS1 optical spectrograph on the COSMOS field (PI F. Fiore). After visual inspection of these eight new galaxies with HST F814W high resolution imaging, none was discarded for evident contamination by foreground objects close to the line of sight of the $z \sim 3.3$ galaxies. The limitation of this procedure, however, is that with only one HST band it is not possible to clearly spot possible blends of multiple sources. We end up with eight additional SFGs in the COSMOS field, which enlarge the original sample adopted by Grazian et al. (2016).

\subsection{EGS field}

In order to improve the significance of our results by increasing the number statistics, we included in the present analysis an additional sample of star-forming galaxies in the Extended Groth Strip (EGS/AEGIS) field (Davis et al. 2007). An area of $\sim 600$ sq. arcmin was covered by two overlapping LBC pointings in the UV band for a total of $6.3 \mathrm{~h}$ (PI H. Yan). The UV depth is 29.6 at a $S / N=1$, with an average seeing of 1.1 arcsec (Grazian, et al., in prep.). The magnitude limit was computed through a PSF-fitting method, as described in the next section. Since no observations in the $R$ band have been acquired by LBC on this specific pointing, we used the $R$-band image taken by the "Deep" program of the CFHT Legacy Survey (hereafter CFHTLS ${ }^{1}$ ). In particular, we used the final release (T0007) of the CFHTLS, which covers 1 sq. deg of the EGS field at a depth of $r=25.6$ at an $80 \%$ completeness level for point sources. The CFHTLS image was resampled to the same WCS astrometric grid of the LBC $U$-band image with $S_{\text {warp }}^{2}$, preserving both the total fluxes of the objects and their original S/N. A large number of spectroscopic redshifts are available in the EGS field from Steidel et al. (2003) and from the DEEP2 survey (Cooper et al. 2006). An initial sample of 16 galaxies was selected with $3.27<z_{\text {spec }}<3.40$. One galaxy (Westphal MD99) was discarded since it was detected at 24 micron by Rigopoulou et al. (2006) suggesting possible AGN activity, and it is also contaminated by a foreground object. Thus, a final sample of 15 galaxies was extracted from the EGS database.

\subsection{CANDELS/GOODS-North field}

In order to explore the LyC radiation of faint galaxies, we successfully proposed an LBT Strategic Program (PI A. Grazian) during the Italian LBT Call for Proposals 2012B, with the aim of obtaining ultra-deep imaging in the $U$ and $R$ bands of the CANDELS/GOODS-North field (Grogin et al. 2011; Koekemoer et al. 2011) with the LBC instrument. The same area has also been observed by other LBT partners (AZ, OSURC, and LBTO), for a total exposure time of $33 \mathrm{~h}$ in $U$ band (seeing $1.1 \mathrm{arcsec}$ ) and $26 \mathrm{~h}$ in $R$ band (seeing $1.0 \mathrm{arcsec}$ ). The detailed description of this dataset is provided in a different paper (Grazian et al., in prep.) summarizing all the LBC deep observations available in the CANDELS fields. The long exposure time and the relatively good seeing obtained allow us to reach a magnitude limit in the $U$ band of $30.2 \mathrm{mag}$ at $S / N=1$, resulting in one of the deepest UV images acquired to date (Ashcraft et al. 2017). The LBC $R$ band, instead, reaches a magnitude limit of 27.8 at $80 \%$ completeness level for point sources.

The GOODS-North field is one of the premier targets of spectroscopic campaigns with the Keck and other smaller telescopes, thanks to the multiwavelength coverage of this area from X-ray to the far-IR. In particular, Barger et al. (2008) collected 2907 spectroscopic redshifts for galaxies and stars in the ACS footprint (Giavalisco et al. 2004), resulting in a $90 \%$ spectroscopic completeness at $B_{435} \leq 24.5 \mathrm{mag}$. Additional information has been provided by Cooper et al. (2011), Pirzkal et al. (2013), and Wirth et al. (2015) just to mention few examples of the numerous works that provide spectroscopic redshifts in the GOODS-North area. We selected from this large database 11 galaxies with $3.27<z_{\text {spec }}<3.40$, the redshift range suitable for LyC analysis with the LBC $U$-band filter. These galaxies are

\footnotetext{
http://www . cfht . hawaii.edu/Science/CFHTLS/

2 http://WwW.astromatic.net/software
} 
Table 1. New galaxies in the COSMOS, EGS, and CANDELS/GOODS-North fields used to derive the LyC relative escape fraction.

\begin{tabular}{|c|c|c|c|c|c|c|c|c|c|}
\hline ID & Field & $\begin{array}{l}\text { RAD } \\
\text { (deg) }\end{array}$ & $\begin{array}{c}\text { Dec } \\
\text { (deg) }\end{array}$ & $z_{\text {spec }}$ & $\begin{array}{c}R \text { mag } \\
(\mathrm{AB})\end{array}$ & $\begin{array}{c}U \text { mag } \\
(\mathrm{AB})\end{array}$ & $M_{1500}$ & $f_{\mathrm{esc}}^{\mathrm{rel}}$ & Cont \\
\hline 53167 & COSMOS & 149.852446 & +2.397032 & 3.359 & 22.95 & $\geq 29.38$ & -22.75 & $\leq 0.029$ & No \\
\hline 218783 & COSMOS & 149.920820 & +2.387060 & 3.297 & 24.49 & $\geq 29.39$ & -21.21 & $\leq 0.118$ & No \\
\hline 223954 & COSMOS & 149.831880 & +2.404150 & 3.371 & 24.77 & $\geq 29.58$ & -20.93 & $\leq 0.128$ & No \\
\hline 219315 & COSMOS & 149.839520 & +2.388460 & 3.363 & 25.21 & $\geq 29.70$ & -20.49 & $\leq 0.171$ & No \\
\hline 215511 & COSMOS & 149.848260 & +2.376170 & 3.363 & 25.24 & $\geq 29.70$ & -20.46 & $\leq 0.176$ & No \\
\hline 220771 & COSMOS & 149.836280 & +2.393190 & 3.359 & 24.84 & $\geq 29.22$ & -20.86 & $\leq 0.188$ & No \\
\hline 217597 & COSMOS & 149.865340 & +2.382770 & 3.284 & 25.73 & $\geq 29.88$ & -19.97 & $\leq 0.233$ & No \\
\hline 211934 & COSMOS & 149.847250 & +2.364040 & 3.355 & 26.86 & $\geq 29.64$ & -18.84 & $\leq 0.827$ & No \\
\hline $59330^{a}$ & COSMOS & 150.077045 & +2.360797 & 3.417 & 24.15 & $\geq 28.93$ & -17.40 & $\leq 0.232$ & No \\
\hline 6328 & EGS & 214.586647 & +52.512559 & 3.357 & 24.26 & $\geq 29.89$ & -21.44 & $\leq 0.060$ & No \\
\hline 9524 & EGS & 214.500676 & +52.477565 & 3.295 & 24.06 & $\geq 29.54$ & -21.64 & $\leq 0.069$ & No \\
\hline 6439 & EGS & 214.552783 & +52.511284 & 3.392 & 24.15 & $\geq 29.56$ & -21.55 & $\leq 0.073$ & No \\
\hline 724 & EGS & 214.501128 & +52.570535 & 3.353 & 24.76 & $\geq 30.06$ & -20.94 & $\leq 0.081$ & No \\
\hline 6922 & EGS & 214.461916 & +52.505537 & & & & -20.99 & & No \\
\hline 4580 & EGS & 214.508258 & +52.531509 & 3.279 & 24.79 & $\geq 29.90$ & -20.91 & $\leq 0.096$ & No \\
\hline 8642 & EGS & 214.460301 & +52.487802 & 3.285 & 24.50 & $\geq 29.60$ & -21.20 & $\leq 0.098$ & No \\
\hline 5197 & EGS & 214.283603 & +52.524388 & 3.271 & 24.52 & $\geq 29.15$ & -21.18 & $\leq 0.150$ & No \\
\hline 6612 & EGS & 214.500033 & +52.509127 & 3.340 & 25.89 & $\geq 30.45$ & -19.81 & $\leq 0.162$ & No \\
\hline 2783 & EGS & 214.554012 & +52.549350 & 3.272 & 25.70 & $\geq 30.02$ & -20.00 & $\leq 0.200$ & No \\
\hline 12335 & EGS & 214.455464 & +52.446348 & 3.287 & 25.32 & $\geq 29.61$ & -20.38 & $\leq 0.205$ & No \\
\hline 13346 & EGS & 214.413697 & +52.433529 & 3.307 & 24.73 & $\geq 28.87$ & -20.97 & $\leq 0.237$ & No \\
\hline 7690 & EGS & 214.249912 & +52.497386 & 3.272 & 25.18 & $\geq 28.77$ & -20.52 & $\leq 0.392$ & No \\
\hline 11372 & EGS & 214.407226 & +52.458201 & 3.294 & 25.36 & 28.48 & -20.34 & 0.603 & No \\
\hline 14043 & EGS & 214.376422 & +52.424380 & 3.344 & 25.18 & $\geq 28.29$ & -20.52 & $\leq 0.610$ & No \\
\hline 16428 & GDN & 189.216181 & +62.254036 & 3.333 & 23.91 & $\geq 29.82$ & -21.79 & $\leq 0.048$ & No \\
\hline 19977 & GDN & 189.179397 & +62.276656 & 3.363 & 24.38 & $\geq 30.03$ & -21.32 & $\leq 0.061$ & No \\
\hline 12305 & GDN & 189.219779 & +62.227532 & 3.369 & 25.09 & $\geq 30.35$ & -20.61 & $\leq 0.087$ & No \\
\hline 4341 & GDN & 189.290579 & +62.171244 & 3.384 & 24.68 & $\geq 29.81$ & -21.02 & $\leq 0.094$ & No \\
\hline 14132 & GDN & 189.206264 & +62.239252 & 3.367 & 25.44 & $\geq 30.23$ & -20.26 & $\leq 0.135$ & No \\
\hline 91200 & GDN & 189.311200 & +62.311020 & 3.390 & 25.76 & $\geq 30.21$ & -19.94 & $\leq 0.184$ & No \\
\hline 16169 & GDN & 189.215365 & +62.252800 & 3.364 & 25.42 & $\geq 29.67$ & -20.28 & $\leq 0.214$ & No \\
\hline 18979 & GDN & 189.296340 & +62.270650 & 3.350 & 25.80 & $\geq 29.90$ & -19.90 & $\leq 0.246$ & No \\
\hline 91000 & GDN & 188.999330 & +62.202417 & 3.300 & 26.53 & $\geq 30.21$ & -19.17 & $\leq 0.377$ & No \\
\hline 16479 & GDN & 189.194532 & +62.254757 & 3.371 & 25.00 & 27.79 & -20.70 & 0.821 & Yes \\
\hline
\end{tabular}

Notes. The galaxies are sorted in ascending order of relative escape fraction in each field (COSMOS, EGS, GDN = CANDELS/GOODS-North). The identification number, ID, is the LBC number for the COSMOS and EGS fields, while it is the CANDELS official identifier for the GOODSNorth field. Two sources, indicated by ID $>90000$, are outside the CANDELS/GOODS-North area. The non-detections (i.e., $S / N<1$ ) in the LBC $U$-band, for which an upper limit (at $1 \sigma$ ) to the relative escape fraction is provided, are indicated by the $U$ mag $\geq$ and $f_{\text {esc }}^{\text {rel }} \leq$ symbols, respectively. The last column, Cont, indicates whether a galaxy is possibly contaminated by foreground objects. Notes on individual objects: (a): object ID = 59330 in COSMOS is the lensed galaxy studied by van der Wel et al. (2013) and by Amorin et al. (2014). The $U$ - and $R$-band magnitudes are the observed photometry for ID $=59330$, while the absolute magnitude provides the intrinsic luminosity after the correction by a magnification factor of $40 \pm 1$.

all the objects in this tiny redshift interval, without any biases against or in favor of Lyman- $\alpha$ emitters. One of these galaxies $(\mathrm{RA}=12: 37: 33.2$, Dec $=+62: 17: 51.9)$ is extremely faint $(R \sim 28)$ and no meaningful constraint on the escape fraction can be achieved with the present depth of the LBC $U$-band image. Moreover, this galaxy is also contaminated by a brighter galaxy at less than 1.5 arcsec distance, so we decided to remove this object from our $z \sim 3.3$ sample. In the end, the final sample in the GOODS-North field consists of ten galaxies, of which two objects (with ID > 90000 in Table 1) are outside the CANDELS area.

\subsection{Faint lensed galaxy sample}

To push our LyC analysis even deeper, we added the lensed galaxy from Amorin et al. (2014), at $z=3.417$ and magnified by a factor of $40 \pm 1$, with intrinsic magnitude of $R_{\text {delensed }}=28.31$, corresponding to $L=0.036 L^{*}\left(M_{1500}=-17.4\right)$. The properties of this galaxy have also been discussed by van der Wel et al. (2013). Interestingly, this galaxy has been classified as a metalpoor galaxy by Amorin et al. (2014), with $12+\log (\mathrm{O} / \mathrm{H})<7.44$ (or equivalently $Z<0.05 Z_{\odot}$ ). In addition, it shows high ionization conditions, with $[\mathrm{OIII}] / \mathrm{H} \beta$ ratio $>5$ and $[\mathrm{OIII}] /[\mathrm{OII}]>10$. 
We also consider two lensed galaxies at $z>3$ with properties similar to this object from the literature (Christensen et al. 2012; Bayliss et al. 2014), where upper limits to the LyC escape fraction have been provided. The properties of these galaxies resemble the Green Peas selected by Cardamone et al. (2009), and they are thus very promising candidates to investigate for their possible contribution to the H I ionizing background, as recently proposed by Izotov et al. (2016a), Vanzella et al. (2016), Schaerer et al. (2016), Stark et al. (2017), and Verhamme et al. (2017).

\subsection{Total sample}

In total, a sample of 79 star-forming galaxies within the redshift range $3.27<z<3.4$ was assembled, of which 45 have already been presented in Boutsia et al. (2011) and Grazian et al. (2016). The properties of the additional 34 galaxies are summarized in Table 1. The results on the LyC emission properties are instead based on the whole sample of these 79 star-forming galaxies with deep LBC data available, and are summarized in the next sections. One of these 79 galaxies is the lens from Amorin et al. (2014). In the final sections, we also consider two additional lensed galaxies at $z \sim 3$ from the literature, without LBC coverage (Christensen et al. 2012; Bayliss et al. 2014). They were added to our sample since they are relatively faint galaxies (magnified by strong lensing) with an independent estimation of their LyC escape fraction.

\section{Method}

\subsection{Estimating the Lyman continuum escape fraction of $z \sim 3.3$ galaxies}

We adopt here the same methodology described by Grazian et al. (2016) in order to estimate the individual values of the galaxy Lyman continuum escape fraction and the sample mean at $z \sim$ 3.3. In particular, we focus on the new sample of 34 galaxies with known spectroscopic redshifts and with wide and deep imaging from COSMOS, EGS, and CANDELS/GOODS-North. Briefly, we summarize here the adopted technique.

Following Grazian et al. (2016), we started from LBC images in the $U$ and $R$ bands, which at $z \sim 3.3$ sample the rest frame wavelengths at 900 and $1500 \AA$, respectively. The relevant galaxies were selected in a narrow redshift range $(3.27<z<3.40)$, with the aim of measuring, with the $U$-band filter of LBC, only the wavelength region at $850 \leq \lambda \leq 900 \AA$ rest frame (see Fig. 1 in Boutsia et al. 2011). The average Lyman continuum absorption by the IGM in the spectra of $z \geq 3$ sources increases rapidly towards bluer wavelengths. For this reason the search for any detection of hydrogen ionizing emission is quite inefficient below $850 \AA$ rest frame, which is sampled by the LBC $U$-band filter in spectra of $z>3.4$ galaxies. The red leakage of the $U$-band filter of $\mathrm{LBC}$ is negligible ${ }^{3}$, and does not affect the following results.

Crucially, almost all the new 34 galaxies have associated deep HST imaging from the COSMOS, EGS, and CANDELS/GOODS-North surveys (Scoville et al. 2007; Grogin et al. 2011; Koekemoer et al. 2011; Stefanon et al. 2017) at several wavelengths from the $V$ to the $H$ bands (for the CANDELS/GOODS-North field the $B$ band of HST is also available), which are essential in order to avoid spurious contamination by foreground sources. In the CANDELS/GOODSNorth field, for example, there are three sources (ID $=16479$,

\footnotetext{
http://lbc.oa-roma.inaf.it/Filters/List.html
}

$16169,18979)$ with bright galaxies with $z_{\text {phot }} \sim 0.7-2.2$ close to their lines of sight. Two $(16169,18979)$ are well separated $(\sim 1.5-1.8$ arcsec $)$, while source ID $=16479$ is closer $(\sim 1.0$ arcsec) and results in a blend in the LBC images. We discuss the properties of this source in the next subsection.

The HST fields used here also have deep X-ray observations by the Chandra satellite (Alexander et al. 2003; Nandra et al. 2015; Civano et al. 2016). We used this information to avoid possible contamination by faint AGNs. None of our 34 galaxies shows any X-ray emission at the levels probed by the Chandra images, thus excluding the presence of an X-ray strong AGN nucleus inside.

As carried out in Grazian et al. (2016), we measured the "relative" escape fraction, which is defined as the fraction of emitted Lyman continuum photons, IGM corrected, related to the observed fraction of photons at $1500 \AA$ rest frame (Steidel et al. 2001; Siana et al. 2007). The relative escape fraction is usually derived from the observations of the flux ratio between 900 and $1500 \AA$ rest frame,

$f_{\mathrm{esc}}^{\mathrm{rel}}=\frac{\left(L_{1500} / L_{900}\right)_{\text {int }}}{\left(F_{R} / F_{U}\right)_{\mathrm{obs}}} \exp \left(\tau_{900}^{\mathrm{IGM}}\right)$,

where $\left(L_{1500} / L_{900}\right)_{\text {int }}$ is the ratio of the intrinsic luminosities at 1500 and $900 \AA$ rest frame, $\left(F_{R} / F_{U}\right)_{\text {obs }}$ is the ratio of the observed fluxes in the $R$ and $U$ bands, and $\tau_{900}^{\mathrm{IGM}}$ is the optical depth of the IGM at $900 \AA$ rest frame. The absolute escape fraction $f_{\text {esc }}^{\text {abs }}$ is defined as $\exp \left(-\tau_{\mathrm{HI}}\right) \times 10^{-0.4 A_{900}}$, where the first factor indicates the ISM extinction, while $A_{900}$ is the dust absorption coefficient at $900 \AA$ rest frame. In this paper, as in Grazian et al. (2016), we adopt a $\left(L_{1500} / L_{900}\right)_{\text {int }}$ value of 3 . In Guaita et al. (2016) we explored the dependencies of this quantity on the various population synthesis models, and concluded that a reasonable range for this ratio is between 2 and 7. In Grazian et al. (2016) we considered the implications of different assumptions of the intrinsic luminosity ratio parameter on the estimations of the escape fraction and LyC emissivity.

To correct for the mean effect of the IGM absorption we adopted the recent estimates by Worseck et al. (2014) and Inoue et al. (2014). Folding the IGM absorption at $z=3.3$ with the $U$-band filter of LBC and adopting the same procedure outlined in Boutsia et al. (2011), we obtain a mean IGM transmission $\left\langle\exp \left(-\tau_{900}^{\mathrm{IGM}}\right)\right\rangle=0.28$ at $z \sim 3.3$. In the next section we explore the implications of the variance of the IGM absorption along multiple lines of sight, using the same methodology adopted in Grazian et al. (2016).

For each galaxy we computed the observed flux ratio $\left(F_{R} / F_{U}\right)_{\text {obs }}$ from the LBC images in the $U$ and $R$ bands in the same way as in Grazian et al. (2016). Briefly, we detected the $z \sim 3.3$ galaxy in the $R$ band using SExtractor (Bertin \& Arnouts 1996), and measured the flux in the $U$ band assuming the same profile of the galaxy in the $R$ band through the ConvPhot software (De Santis et al. 2007), taking into account the transfer function (convolution kernel) to change the spatial resolution of the $R$ band to $U$ band. As discussed in Grazian et al. (2016), this PSF-matched photometry allows us to derive errors on fluxes which are typically a factor of 1.7 smaller than those measured by SExtractor through aperture photometry. In the case of nondetection in the $U$ band, a $1 \sigma$ upper limit to the flux in this filter is set by the flux error provided by ConvPhot. Assuming an average IGM transmission of 0.28 at $z \sim 3.3$, we compute the relative escape fraction for each individual galaxy adopting Eq. (1), and provide the relevant quantities in Table 1 . 

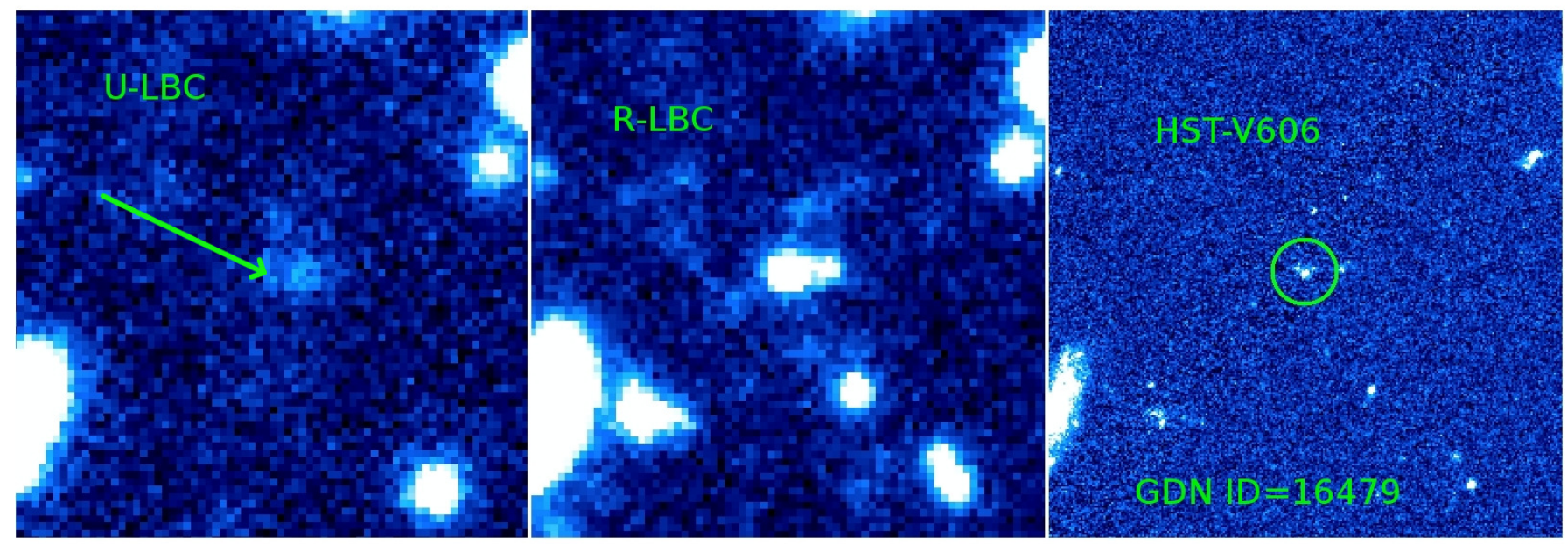

Fig. 1. Cutouts of source ID $=16479$ of the CANDELS/GOODS-North field in the $U$ band (left) and in the $R$ band (center) observed by the LBC instrument. The cutout on the right shows the same sky area observed by HST in the V606 filter. The position of the galaxy with spectroscopic redshift at $z=3.371$ is indicated by the green circle. The green arrow in the $U$ band marks the position of the centroid of source ID $=16479$ derived from the $R$-band image. At $\sim 1.0$ arcsec from the $z \sim 3.3$ source, towards the right, there is a faint galaxy at $z_{\text {phot }}=2.2$ that is clearly detected in all three bands. Close to the main target there are also two very faint blobs in the HST image, which are blended with the $z \sim 3.3$ galaxy in the ground-based LBC images. The size of each image is 17 arcsec by 17 arcsec.

\subsection{Detailed analysis of the possible LyC emitters}

In Grazian et al. (2016) we discussed the issue of foreground contaminants mimicking significant LyC detection in the COSMOS field. In Table 1 there are two galaxies with significant LyC relative escape fraction $(\sim 60-80 \%)$, ID $=11372$ in the EGS field and ID = 16479 in GOODS-North. In order to determine their reliability, we carried out some checks, as done in Grazian et al. (2016). In particular, we used HST data from the CANDELS and AEGIS surveys in order to evaluate the reliability of these LyC emitter candidates.

Figure 1 shows the galaxy ID $=16479$ in the CANDELS/GOODS-North field in the $U$ and $R$ band observed by LBC and in the HST V606 filter (the closest band to the LBC- $R$ filter). At the right of the $z=3.371$ galaxy (identified by a green circle in the HST thumbnail) there is a faint source at $\sim 1.0$ arcsec distance, which is clearly detected in the three bands. If we assume that the two sources are at the same redshift $(z=3.371)$ and compute their escape fraction, we find $f_{\mathrm{esc}}^{\mathrm{rel}}=206 \%$. If we evaluate the escape fraction only of the fainter galaxy to the right (outside the green circle) and assume that it is at $z \sim 3.3$, we end up with $f_{\text {esc }}^{\text {rel }}=627 \%$. In this case, as already reported by Vanzella et al. (2012a) and Grazian et al. (2016), the so-called "local" escape fraction (i.e., calculated in a small region showing LyC emission) is not compatible with the stellar population of a $z \sim 3.3$ galaxy, indicating a clear sign of contamination by a lower- $z$ interloper. In fact, even assuming a higher ionizing efficiency (i.e., a $\left(L_{1500} / L_{900}\right)_{\text {int }}$ value of 2.0, instead of 3.0) and a more transparent line of sight with $\exp \left(-\tau_{\text {IGM }}\right)=0.68$ (i.e., the upper value of the $z \sim 3.3$ IGM transmission at $95 \%$ confidence level), the resulting local escape fraction is $170 \%$, confirming the interloper interpretation for this object. Indeed, the brighter galaxy at the center of the green circle in Fig. 1 has different colors from the faint blob on the right, which is thus probably at a different redshift. This system has already been studied by Reddy et al. (2006), labeled as BX1334. Interestingly, it has been selected as a BX galaxy (with colors compatible with galaxies at photometric redshifts between 2.0 and 2.7; see Adelberger et al. 2004), and it has
near-IR colors compatible with the distant red galaxy (DRG) criterion $(R-K \geq 1.3 \mathrm{mag}, z \sim 2$ according to Franx et al. 2003). From the CANDELS photometry a redshift of $z_{\text {phot }}=2.2$ has been derived for this close-by source, indicating that this is plausibly a lower- $z$ contaminant.

Using HST data in the V606 band, it is possible to compute the relative escape fraction of the $z \sim 3.3$ galaxy reducing the contamination by the foreground interloper on the right. Adopting as a prior for ConvPhot the HST image we obtain a $U$-band magnitude of 27.79 , corresponding to $f_{\mathrm{esc}}^{\mathrm{rel}}=82 \%$. This could thus be a genuine LyC emitter at $z \sim 3.3$. However, close to the $z \sim 3.3$ galaxy there are also two very faint blobs in the HST image, which are blended with the main target in the ground-based LBC images. A faint off-center emission in the $U$ band of LBC has been detected, and this is possibly associated with the faint blob close to the $z=3.371$ galaxy (towards the right). There is thus an indication of a possible contamination by foreground objects, even if in this case no firm conclusions can be drawn.

The second object ID $=11372$, shown in Fig. 2, is at best marginally detected at $1.5 \sigma$ in the $U$ band, and its relative escape fraction is $f_{\mathrm{esc}}^{\mathrm{rel}} \sim 60.3 \%$. This galaxy was originally studied by Steidel et al. (2003) as the object C029 of the Westphal field. Its morphology in the HST V606 band is almost point-like, but no X-ray detection has been found for this galaxy, and thus an AGN classification for this object cannot be confirmed. Even for this object there is a hint in the HST image of a very faint blob close to the $z \sim 3.3$ galaxy (at $\sim 0.1$ arcsec towards the right), which can act as a possible faint contaminant, compatible with the marginal $(1.5 \sigma)$ detection in the $U$ band. However, no firm conclusions can be drawn for this galaxy, and deeper data from LBC in the $U$ band and HST in the V606 band are needed in order to understand its nature. It is possible that the distribution of the escape fraction of $z>3$ galaxies is bimodal, with the bulk of the star-forming population showing small values of LyC emission, while few objects emit large amounts of ionizing radiation (e.g., Ion2, Vanzella et al. 2016).

In Table 1 , we mark the ID = 16479 galaxy as possibly contaminated by a foreground object, while the galaxy ID $=11372$, where this hypothesis is weak, is considered as a possible LyC 

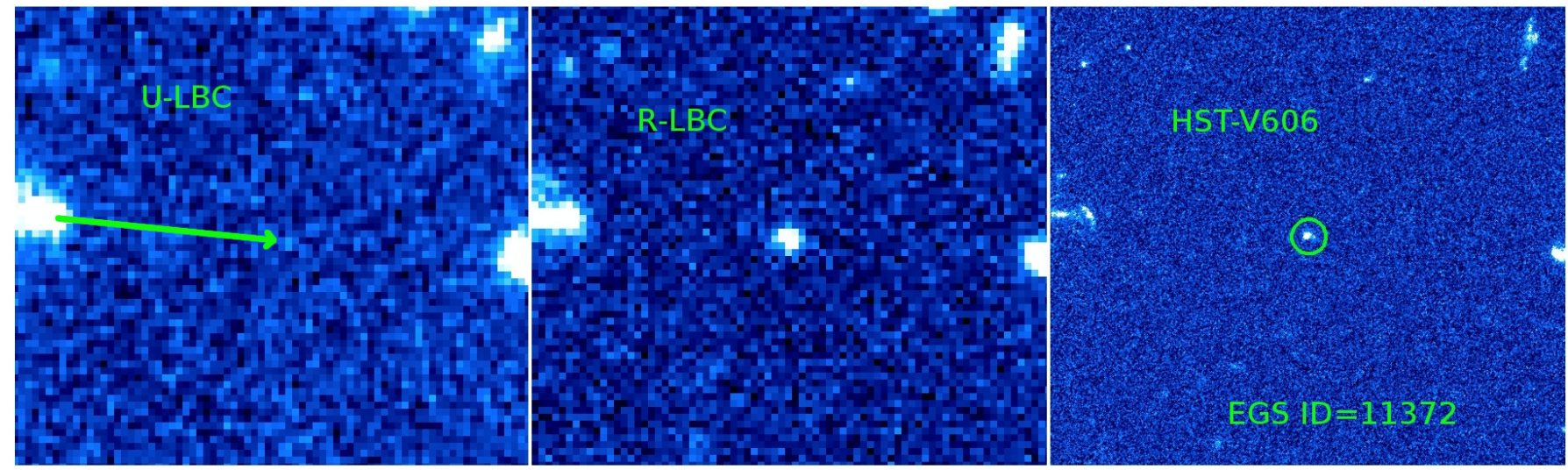

Fig. 2. Cutouts of source ID $=11372$ of the EGS field in the $U$ band (left) and in the $R$ band (center) observed by the LBC instrument. The cutout on the right shows the same sky area observed by HST in the $V 606$ filter. The position of the galaxy with spectroscopic redshift at $z=3.294$ is indicated by the green circle. The green arrow in the $U$ band marks the position of the centroid of source ID $=11372$ derived from the $R$-band image. In the LBC $U$ band there is a marginal detection $(1.5 \sigma)$. The size of each image is $17 \operatorname{arcsec}$ by $17 \operatorname{arcsec}$.

emitter, pending deeper observations. In the following sections we investigate the properties of the whole star-forming galaxy population at $z \sim 3.3$, relying on the stacking procedure of the LBC images.

In summary, the properties of the new sample of 34 galaxies at $z \sim 3.3$ in the COSMOS, EGS, and CANDELS/GOODSNorth fields are listed in Table 1 . They were merged with the 45 SFGs at the same redshifts studied in Grazian et al. (2016) from the VUDS/LBC-COSMOS database, resulting in a total sample of 79 galaxies. However, not all of them have been used to compute the H I ionizing emissivity of the SFG population at $z \sim 3$. We discarded nine galaxies possibly affected by contamination due to foreground objects. Of this subsample, eight galaxies were discussed in detail in Grazian et al. (2016) and an additional galaxy has been discarded from Table 1, i.e., ID $=16479$ in the CANDELS/GOODS-North field. The galaxy ID = 59330 in the COSMOS field is the lensed object studied by Amorin et al. (2014) and it is not considered in the stacking procedure, which is restricted to the 69 galaxies of luminosity $L \gtrsim 0.2 L^{*}$.

\section{Results}

We consider here the stack of the cleaned sample in the $U$ and $R$ bands by LBC and provide the mean $f_{\mathrm{esc}}^{\text {rel }}$ values for the whole population of 69 galaxies not affected by contamination and for various subsamples at different $R$-band magnitudes.

Figure 3 summarizes the $f_{\text {esc }}^{\text {rel }}$ properties of the cleaned sample as a function of the observed $R$-band magnitude. In this plot, the blue symbols indicate the VUDS/LBC-COSMOS galaxies published in Grazian et al. (2016), while the green, red, and gray arrows are for sources in the COSMOS (Onodera et al. 2016), CANDELS/GOODS-North, and EGS fields, respectively. The small downward arrows indicate $1 \sigma$ upper limits for the individual $f_{\text {esc }}^{\text {rel }}$, while asterisks show detections in LyC above $1 \sigma$. The red asterisk in the hexagon indicates the galaxy ID $=16479$ in the CANDELS/GOODS-North field, which is probably affected by foreground contamination. The gray, blue, and red dashed lines show the escape fraction versus $R$-band magnitude corresponding to the $1 \sigma$ depth of the $U$ band in the EGS, COSMOS, and CANDELS/GOODS-North fields, respectively $(U=$ 29.6, 29.7, and 30.2), derived using Eq. (1). The scatter of the observed galaxies around these lines is due to the small differences in depth on the images and on the different morphologies of the sources (more extended galaxies have slightly shallower magnitude limits).

\subsection{Constraints on the escape fraction from stacking}

We then proceed with the stacking of the LBC $U$ and $R$ bands of the clean sample in different luminosity intervals. We repeat the same procedures outlined in Grazian et al. (2016), which we summarize in the following. We cut a small thumbnail $\left(\sim 45^{\prime \prime} \times 45^{\prime \prime}\right)$ of the LBC $U$ - and $R$-band images centered on each $z \sim 3.3$ galaxy; we mask all the nearby sources detected in the $R$ band, leaving the central region unmasked; and then we compute the weighted mean of all the thumbnails in a given band using the inverse variance map as a weight. We measure the $f_{\text {esc }}^{\text {rel }}$ on the stacked images adopting the same technique outlined above, i.e., using ConvPhot.

We first proceed with the stacking in different $R$-band magnitude intervals, then we carry out the sum of all the galaxies in Grazian et al. (2016) and in Table 1, except for the contaminated object $(\mathrm{ID}=16479)$ in the CANDELS/GOODS-North field and the lensed galaxy from Amorin et al. (2014). We do not achieve any significant detection in the $U$-band above $1 \sigma$ in the stacked images, resulting in several upper limits to the LyC escape fraction. We plot the results of the stacking in different luminosity intervals in Fig. 3 with large arrows, and summarize all the relevant values in Table 2 .

We do not detect for the stacks significant dependencies of $f_{\mathrm{esc}}^{\mathrm{rel}}$ on luminosity at $R \leq 25.5$, corresponding to $L>0.5 L^{*}$, where a constant value of $2-2.5 \%$ has been determined (cyan, purple, and orange arrows in Fig. 3). Stacking of all 69 galaxies (we do not consider the lensed galaxy by Amorin et al. 2014) in $U$ and $R$ band resulted in a non-detection in the $U$ band to a level of $U=31.74$ at $1 \sigma$ and a clear detection in the $R$ band of $R=24.73$ at $S / N=414$, as shown in Fig. 4. This translates into a limit to the observed ratio between $R$ and $U$ band of $F_{R} / F_{U} \geq 640.2$, which is equivalent to an upper limit to the relative escape fraction of $f_{\mathrm{esc}}^{\mathrm{rel}} \leq 1.7 \%$ at $1 \sigma$ level at $z=3.3$ (magenta arrow in Fig. 3). This is the most stringent limit to the relative escape fraction for $z>3$ star-forming galaxies. The inclusion of the three galaxies detected in the $U$ band (two galaxies discussed in Grazian et al. (2016) and ID = 16479 in the CANDELS/GOODS-North field) does not significantly change the results of the stacking $\left(f_{\mathrm{esc}}^{\mathrm{rel}} \leq 1.8 \%\right)$. 


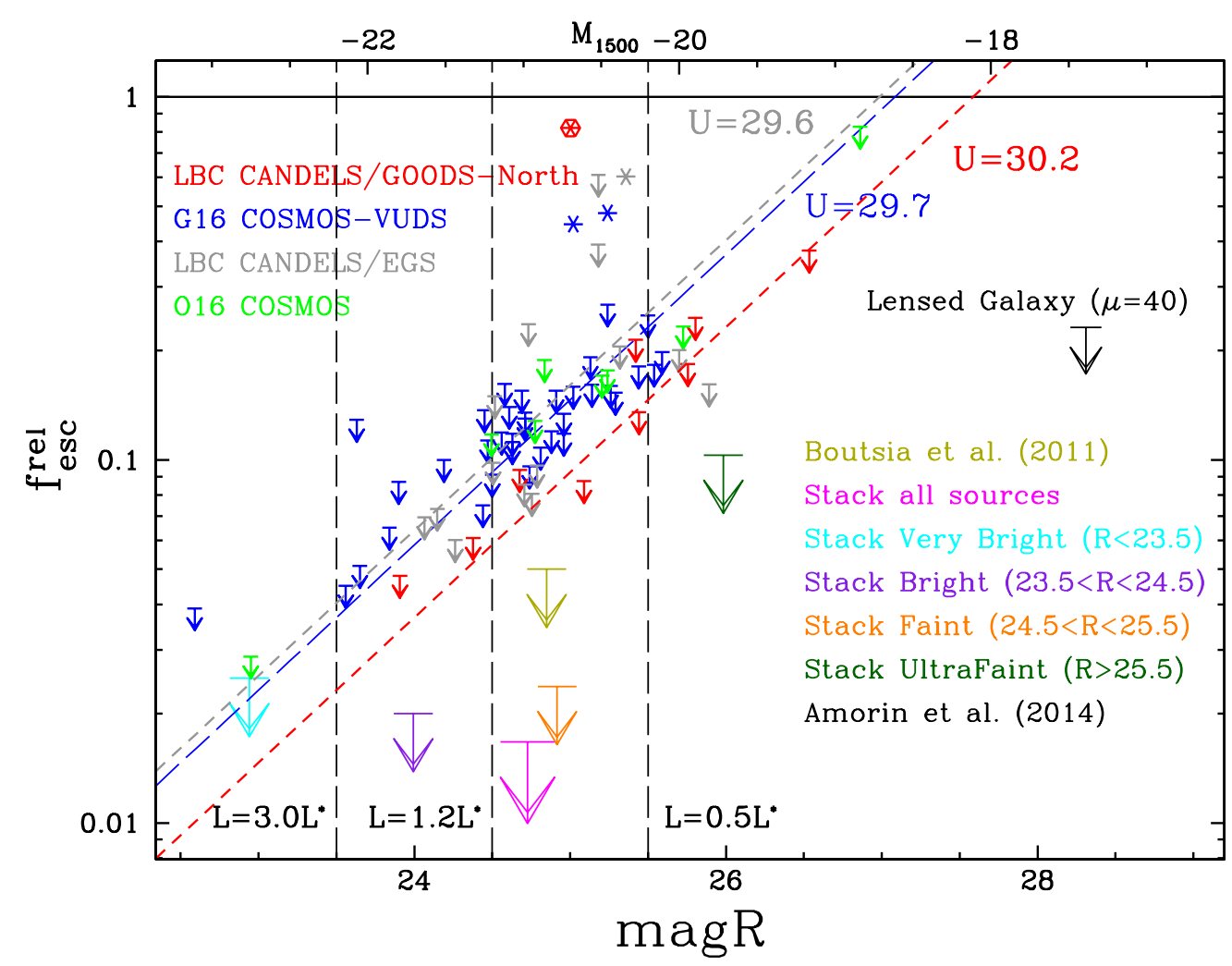

Fig. 3. Measured values and the upper limits at $1 \sigma$ for the LyC relative escape fraction of galaxies at $z \sim 3.3$ in the COSMOS (blue, green), EGS (gray), and CANDELS/GOODS-North fields (red). Small descending arrows show the upper limits of $f_{\text {esc }}^{\text {rel }}$ associated with individual galaxies. Asterisks show the galaxies detected in the $U$ band (i.e., LyC emission) above $1 \sigma$. The red hexagon indicates a likely foreground contamination in the CANDELS/GOODS-North field, associated with galaxy ID = 16479. The lensed galaxy from Amorin et al. (2014), magnified by a factor $\mu=40 \pm 1$, is represented by a black arrow at its intrinsic (de-magnified) magnitude $R=28.31$. The gray, blue, and red dashed lines show the dependency of the escape fraction on the $R$-band magnitude derived by adopting the $1 \sigma$ depth of the $U$ band in the EGS, COSMOS, and CANDELS/GOODS-North fields, respectively. The large arrows (cyan, purple, magenta, orange, gold, dark green) represent the limits to $f_{\text {esc }}^{\text {rel }}$ derived by stacking the $z \sim 3.3$ galaxies from Grazian et al. (2016) and from this paper in different $R$-band magnitude intervals.

Table 2. Escape fraction of stacks.

\begin{tabular}{ccccc}
\hline \hline Sample & $N_{\text {gal }}$ & $\begin{array}{c}R \text { mag } \\
(\mathrm{AB})\end{array}$ & $M_{1500}$ & $\begin{array}{c}f_{\text {esc }}^{\text {rel }} \\
(1 \sigma)\end{array}$ \\
\hline Very bright $(R \leq 23.5)$ & 2 & 22.94 & -22.8 & $\leq 0.025$ \\
Bright $(23.5<R \leq 24.5)$ & 16 & 23.99 & -21.7 & $\leq 0.020$ \\
Faint $(24.5<R \leq 25.5)$ & 42 & 24.92 & -20.8 & $\leq 0.024$ \\
Ultra-faint $(R>25.5)$ & 9 & 25.98 & -19.7 & $\leq 0.103$ \\
\hline Stack all sources & 69 & 24.73 & -21.0 & $\leq 0.017$ \\
\hline Lensed $\left(R_{\text {delensed }=28.31)}\right.$ & 1 & 28.31 & -17.4 & $\leq 0.232$ \\
\hline Christensen et al. (2012) & 1 & & -20.4 & $\leq 0.11$ \\
Bayliss et al. (2014) & 1 & & -20.5 & $\leq 0.01$ \\
\hline
\end{tabular}

Notes. The lensed galaxy with $R_{\text {delensed }}=28.31$ is described in Amorin et al. (2014). The galaxy from Bayliss et al. (2014) has $\log N(\mathrm{HI}) \geq 21.5$, resulting in a null escape fraction. We provide here a very conservative upper limit of $1 \%$ for this object.

The uncertainties on the relative escape fraction of the stack due to the IGM variance was derived following the same procedures outlined in Grazian et al. (2016). Considering 69 lines of sight, the mean IGM transmission, folded with the $U$-band filter of LBC, has an uncertainty of $\sigma=0.023$. Using this error, the $68 \%$ confidence level for our value of $f_{\mathrm{esc}}^{\mathrm{rel}}$ is between 1.54 and $1.82 \%$. In the following, we use the derived limit $f_{\mathrm{esc}}^{\mathrm{rel}} \leq 1.7 \%$ without considering its small associated uncertainty.

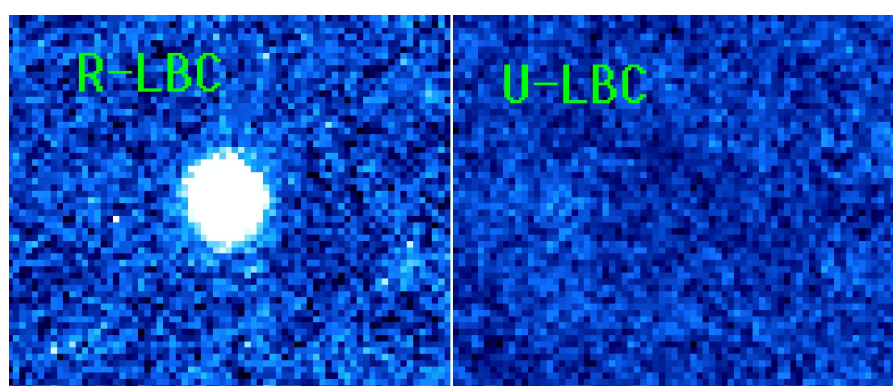

Fig. 4. Stack of the 69 galaxies with $3.27 \leq z_{\text {spec }} \leq 3.40$ in the $R$ (left) and $U$ bands (right) for the COSMOS, EGS, and CANDELS/GOODSNorth fields. The size of each image is 16 arcsec by 14 arcsec.

\subsection{Constraints on the escape fraction from strong gravitational lensing}

Ultra-deep UV imaging in blank fields with LBC reach a magnitude limit of $\sim 30$ (AB), but these depths do not allow us to push the constraints of the escape fraction below $\sim 10 \%$ at $M_{1500} \gtrsim-20$. On the other hand, relatively deep LBC data in the $U$ and $R$ bands have been used to put a limit to the LyC escape fraction of the lensed galaxy by Amorin et al. (2014), thus allowing us to explore the regime of sub- $L^{*}$ galaxies at $z>3$. A $1 \sigma$ upper limit to the escape fraction of $23.2 \%$ has been derived at $M_{1500}=-17.4$, which can add new observational constraints to the theoretical models predicting that sub- $L^{*}$ galaxies at high- $z$ 
have high escape fractions, and are thus the main drivers of the reionization of the Universe (e.g., Ferrara \& Loeb 2013; Ellis 2014; Bouwens et al. 2015; Finkelstein et al. 2015).

Other examples of strongly magnified galaxies at $z \gtrsim 3$ can be found in the literature. Christensen et al. (2012) reported the case of a lensed LBG (SMACS J2031.8-4036 ID 1.1) at $z \sim 3.5$ with a LyC escape fraction estimate from their X-shooter spectrum. This galaxy has an absolute magnitude after correction for magnification of $M_{1500}=-20.4$, and the constraint on the escape fraction is $f_{\text {esc }}^{\mathrm{rel}} \leq 3-11 \%$. Their conservative upper limit for the escape fraction, thanks to strong lensing magnification, is fully consistent with the individual limits on $z \sim 3.3$ galaxies at the depth of the CANDELS/GOODS-North $U$-band observations of this paper. The spectral properties of this peculiar galaxy are similar to those observed in Ion2 by Vanzella et al. (2016), and usually associated with possible LyC emissivity; this galaxy indeed shows low metallicity $(Z=$ $\left.0.1 Z_{\odot}\right)$ and a high ionization parameter $(\log U=-2.1)$, presenting high $[\mathrm{OIII}] /[\mathrm{OII}]$ and $[\mathrm{OIII}] / \mathrm{H} \beta$ line ratios. Following recent claims (Vanzella et al. 2016; Izotov et al. 2016b; Schaerer et al. 2016; Nakajima \& Ouchi 2014; Nakajima et al. 2016), a much larger escape fraction should be expected for this object. Owing to its bright observed magnitude $\left(V_{606}=21.19 \pm 0.10\right)$ this galaxy could be an ideal target for future deep follow-up spectroscopy with efficient UV spectrographs in the LyC region.

Another interesting object is the Green Pea SGASJ105039.6+001730 at $z=3.6252$ studied by Bayliss et al. (2014). This galaxy has a magnification factor of $\mu \geq 30$, with an absolute magnitude of $M_{1500}=-20.5$ after corrections for strong lensing. Like the previous example, this object also shows line ratios of $[\mathrm{OIII}] /[\mathrm{OII}]>10$ and $[\mathrm{OIII}] / \mathrm{H} \beta>10$, and is expected to emit ionizing radiation with a large escape fraction, due to the significant ionization efficiency. Surprisingly, the spectroscopic data indicate that the neutral column density is $\log N_{\mathrm{HI}}>21.5$ and thus the escape fraction is negligible $\left(f_{\mathrm{esc}}^{\mathrm{rel}}<1 \%\right.$ ). It is worth noting that the profile of the Lyman- $\alpha$ line in emission is also compatible with an associated absorption, in agreement with the measured high column density of neutral hydrogen. If its low $f_{\mathrm{esc}}^{\text {rel }}$ is confirmed, this result gives stringent limits to the LyC escape fraction at $z \geq 3$, which are comparable with the constraints we obtain in this work by stacking 69 galaxies $(\leq 1.7 \%)$. It thus stresses the importance of the lensing magnification for this type of study, as also shown by Vasei et al. (2016) at $z \sim 2$. Similar studies will be important in the future to extend the investigated range of $f_{\mathrm{esc}}^{\text {rel }}$ at very low luminosities.

\subsection{Does the escape fraction depend on luminosity?}

In Grazian et al. (2016) we showed that star-forming galaxies brighter than $0.5 L^{*}$ at $z \sim 3$ cannot produce the UV background (UVB) as measured using the Lyman forest. Here we explore the role of fainter galaxies.

In Fig. 3 we observe an apparent trend of looser limits of the escape fraction with fainter $R$-band magnitudes. This correlation is clearly due to the $U$-band depth of our imaging database. At a given luminosity, the measured upper limits on the escape fraction of each individual galaxy actually depend on the different depths of the COSMOS, EGS, and CANDELS/GOODSNorth fields, and they are not related to an intrinsic property in the population of star-forming galaxies.
The stacking at $R \leq 25.5$ indicates that $f_{\text {esc }}^{\text {rel }}$ is below the $\sim 2-2.5 \%$ level, while at fainter magnitudes the constraints are less well determined, with $f_{\mathrm{esc}}^{\mathrm{rel}} \leq 10 \%$ at $25.5 \leq R \leq 27.5$ and $f_{\text {esc }}^{\text {rel }} \leq 23 \%$ at $R \sim 28.3 \mathrm{mag}$, even if in the latter case only one galaxy has been used to provide this limit, and uncertainties due to the IGM variance still hold.

Leethochawalit et al. (2016), using the gas covering fraction $\left(f_{\text {cov }}\right)$ of low ionization gas as an indirect proxy for the galaxy escape fraction ( $f_{\text {esc }}^{\text {abs }} \sim 1-f_{\text {cov }}$ neglecting dust absorption), found that there is a weak anti-correlation of the supposed $f_{\text {esc }}^{\text {rel with }}$ the SFR, with a typical value of $f_{\mathrm{esc}}^{\mathrm{rel}} \sim 20 \%$ for SFR on the order of $10-100 M_{\odot} / \mathrm{yr}$ at $4<z<5$. The range in SFR of their galaxies translates into an $R$-band magnitude of $R \sim 25-28$, so it is interesting to note that the value of $f_{\mathrm{esc}}^{\mathrm{rel}}$ inferred in their work is slightly higher than what we measure.

A rough explanation is that the gas covering fraction can only provide an upper limit to the LyC escape fraction, which in fact could be significantly lower. A notable example is the lensed galaxy by Vasei et al. (2016): it has a low gas covering fraction, mimicking an escape fraction of $f_{\mathrm{esc}}^{\text {rel }} \sim 40 \%$, but when a direct measurement is carried out, a $3 \sigma$ upper limit of $8 \%$ is derived, significantly lower than the value inferred from the gas covering factor (see also Dijkstra et al. 2016). These comparisons point thus to a possible inconsistency between the indirect results derived from the low covering fraction and the direct measurements of the LyC escape fraction. It is thus desirable that future claims of significant LyC emission be substantiated by direct detections. However, it is useful to recall here that the limits by Amorin et al. (2014) or Vasei et al. (2016) are based on single non-detections, which could also be due to a line of sight with substantial IGM attenuation, or to a patchy ISM. Larger databases of lensed, intrinsically faint galaxies are needed to distinguish these alternative hypotheses.

The data in Table 2 are summarized in Fig. 5. Black arrows are the limits derived in this paper with LBC data through stacking, while blue arrows report constraints of individual lensed galaxies from the literature (Christensen et al. 2012; Amorin et al. 2014; Bayliss et al. 2014). We explore here different dependencies of the relative escape fraction with luminosity, with the aim of computing the total ionizing emissivity of faint galaxies. The galaxy from Bayliss et al. (2014) has $\log N(\mathrm{HI}) \geq 21.5$, resulting in a very small escape fraction $\left(f_{\text {esc }}^{\text {rel }}<1 \%\right)$. We provide here a very conservative upper limit of $1 \%$ for this galaxy in Fig. 5. The real emissivity value will be probably lower than this limit.

Different parameterizations of the escape fraction as a function of luminosity are explored in Fig. 5. It is worth stressing here that these dependencies are not the best fits to the observed data; they are simply analytic functions (constant, exponential or power law) that are reasonably consistent with the observed stack limits, i.e., with looser constraints at fainter luminosities. These functions are not physically motivated, but they explore possible dependencies of the escape fraction on luminosity $f_{\text {esc }}^{\text {rel }}(L)$ allowed by the observed constraints. Indeed, it is useful to note that the contribution of bright galaxies does not change significantly if we impose $f_{\mathrm{esc}}^{\text {rel }}<1 \%$ or $f_{\mathrm{esc}}^{\text {rel }}<2 \%$, due to the exponential cut-off of the luminosity function at the bright end.

In Fig. 5 we explore five different parameterizations for $f_{\mathrm{esc}}^{\mathrm{rel}}(L)$ :

- A: constant $f_{\text {esc }}^{\text {rel }}$ at $1.7 \%$ at all luminosities (continuous red line), not consistent with the limit of Bayliss et al. (2014), but easily scalable to $f_{\mathrm{esc}}^{\mathrm{rel}} \leq 1 \%$ with a simple multiplicative factor; 


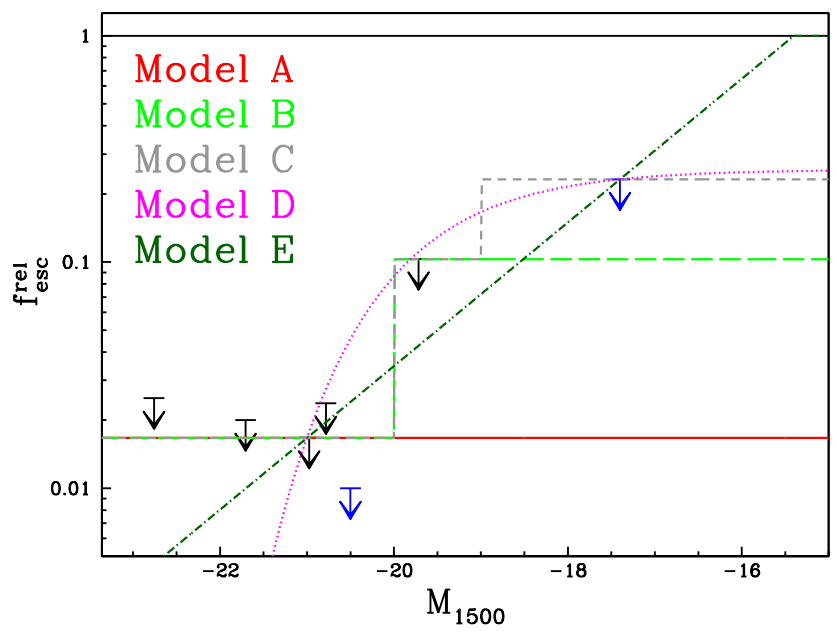

Fig. 5. The dependence of the relative escape fraction from the absolute magnitude in the UV $M_{1500}$. The black arrows are the upper limits to the relative escape fractions derived from the stacks of LBC data in this paper, summarized in Table 2. The observed trend of looser upper limits with fainter magnitudes is due to the limited depth of the data in the UV wavelengths, and plausibly it is not related to a physical property of the population of star-forming galaxies. The blue arrows summarized the values available in literature for individual lensed galaxies, by Amorin et al. (2014) and Bayliss et al. (2014), in the luminosity ranges not covered by our data. For the Bayliss et al. (2014) source, the limit of $1 \%$ has been plotted only for graphical reasons, but could be significantly lower. The different curves show analytic functions which have been adopted to infer the evolution of the relative escape fraction from luminosity. These functions have been used in order to check for the contribution of galaxies at different luminosities to the observed UVB.

- B: two-step function of $1.7 \%$ at $M_{1500} \leq-20.0$ and $10.3 \%$ at fainter magnitudes (long-dashed green line);

- C: three step function of $1.7 \%$ at $M_{1500} \leq-20.0,10.3 \%$ at $-20.0<M_{1500} \leq-19.0$, and $23.2 \%$ at fainter magnitudes (short-dashed gray line);

- D: exponential law $f_{\text {esc }}^{\text {rel }}=f_{1} \times \exp (-\alpha \cdot L / L *)$ with $f_{1}=0.256$ and $\alpha=2.7$ (dotted magenta line);

- E: power law $f_{\mathrm{esc}}^{\mathrm{rel}}=f_{1} \cdot(L / L *)^{-\alpha}$ with $f_{1}=0.0167$ and $\alpha=0.79$ (dot-dashed dark green line).

The various evolutionary parameterizations of the escape fraction as a function of the galaxy luminosity are used in the next section to derive different estimates of the average galaxy emissivity at $z \sim 3.3$.

\section{Estimating the ionizing emissivity produced by star-forming galaxies at $z \sim 3$}

The rate of ionizing photons produced by star-forming galaxies can be expressed as $\dot{n}_{\text {ion }}=\rho_{\mathrm{UV}} \cdot \xi_{\text {ion }} \cdot f_{\text {esc }}^{\text {abs }}$, where the three terms on the right side can be evaluated upon reasonable assumptions.

The first term, $\rho_{\mathrm{UV}}$, is the emissivity of high- $z$ star-forming galaxies, measured at non-ionizing wavelengths, typically at $1500 \AA$ rest frame. It can be obtained by integrating the luminosity function of galaxies, multiplied by their luminosities, down to faint limits, e.g., $L \sim 10^{-3}-10^{-2} L^{*}$. The ionization efficiency $\xi_{\text {ion }}$ measures the rate of ionizing photons produced by a given amount of UV luminosity (at $1500 \AA$ rest frame). It depends on the nature of the stellar populations that developed in distant galaxies (i.e., IMF, age, metallicity, binary fraction). Finally, the absolute escape fraction $f_{\mathrm{esc}}^{\text {abs }}$ measures the percentage of ionizing radiation produced by stars that is not absorbed by gas and dust in the ISM and is finally emitted in the surrounding IGM. In practice, it is the ratio between the observed and produced fluxes at $900 \AA$ rest frame. Numerous attempts have been carried out recently to evaluate the ionization efficiency $\xi_{\text {ion }}$ of high-z galaxies (e.g., Dunlop et al. 2013; Robertson et al. 2013; Bouwens et al. 2016a).

However, a number of assumptions are hidden in the calculations of the rate of ionizing photons $\dot{n}_{\text {ion }}$, and its derivation suffers from intrinsic degeneracies between the physical properties assumed for the underlying stellar populations. More importantly, the two quantities $\xi_{\text {ion }}$ and $f_{\text {esc }}^{\text {abs }}$ are by definition degenerate since different combinations of absolute escape fraction and production efficiency of ionizing photons can give the same observed properties for a galaxy. It is actually not easy to provide an unbiased estimation for the absolute escape fraction $f_{\mathrm{esc}}^{\mathrm{abs}}$. Since the intrinsic SEDs of star-forming galaxies are not known a priori in the far UV, assumptions on the physical properties of the galaxies (stellar populations, age, star formation history, metallicity, etc.) must be adopted. In particular, the choice of stellar population synthesis model can change the intrinsic LyC emissivity up to a factor of 1.5 or 2, if prescriptions for the treatment of the stellar binaries are adopted (e.g., Eldridge \& Stanway 2009; Stanway et al. 2016). Moreover, since the ages of our galaxies are not well constrained, the intrinsic LyC production has a similar level of uncertainty.

We use, instead, an alternative approach based on the relative escape fraction, as described in Eq. (1). Following Grazian et al. (2016), we chose to derive the ionizing emissivity adopting the relation

$$
\rho_{900}^{\mathrm{esc}}=\rho_{1500} \cdot f_{\mathrm{esc}}^{\mathrm{rel}} \cdot\left(L_{900} / L_{1500}\right)_{\mathrm{int}},
$$

where the factor $\left(L_{900} / L_{1500}\right)_{\text {int }}$ is proportional to $\xi_{\text {ion }}$. In this case the advantage, thanks to Eq. $(1)$, is that $f_{\mathrm{esc}}^{\mathrm{rel}} \cdot\left(L_{900} / L_{1500}\right)_{\text {int }}$ can be written as $\left(F_{U} / F_{R}\right)_{\text {obs }} \exp \left(\tau_{\mathrm{IGM}}\right)$ which is the product of two quantities less subject to systematic uncertainties or assumptions, and neglecting the extinction by dust it is equivalent to $\xi_{\text {ion }} \cdot f_{\text {esc }}^{\text {abs }}$.

The "relative" escape fraction, by definition, depends on the assumed ratio $\left(L_{1500} / L_{900}\right)_{\text {int }}$ and as such is not free from assumptions on the physical properties of the galaxies. But its main advantage, as discussed in Grazian et al. (2016), is that its dependencies on the physical properties of the galaxies are canceled out when the emissivity is computed at $\lambda \leq 900 \AA$ rest by combining Eq. (1) with Eq. (2). The only parameter critically affecting Eq. (2) is the input luminosity density of non-ionizing radiation, $\rho_{1500}$. To obtain this value it is sufficient to integrate an observed luminosity function, multiplied by the absolute luminosity, down to a specific magnitude limit.

In addition, it is useful to express the LyC emissivity $\rho_{900}^{\text {esc }}$ in terms of the $\mathrm{H}$ I photoionizing background $\Gamma_{-12}$, in units of $10^{-12}$ photons per second. Following Grazian et al. (2016), we have

$\Gamma_{-12}=\frac{10^{12} \cdot \rho_{900}^{\mathrm{esc}} \cdot \sigma_{\mathrm{HI}} \cdot \Delta l \cdot(1+z)^{3}}{h_{P} \cdot\left(3+\left|\alpha_{\mathrm{UV}}\right|\right)}$,

where $\sigma_{\mathrm{HI}}=6.3 \times 10^{-18} \mathrm{~cm}^{2}$ is the photoionization cross section of hydrogen at $\lambda=912 \AA, \alpha_{\mathrm{UV}}=-1.8$ is the spectral slope of the stellar ionizing radiation (see Faucher-Giguère et al. 2009), and $h_{\mathrm{P}}$ is the Planck constant. The mean free path of ionizing photons $\Delta l$ is 83.5 proper Mpc at $z=3.3$, following the fitting formula by Worseck et al. (2014). 
A. Grazian et al.: Lyman continuum escape fraction of galaxies at $z=3.3$

Table 3. Schechter coefficients of the luminosity function of star-forming galaxies at $z \sim 3$.

\begin{tabular}{cccccc}
\hline \hline LF & $M^{*}$ & $\Phi^{*}$ & $\alpha$ & Reference & Notes \\
\hline 01 & -20.71 & 0.00055 & -1.94 & Alavi et al. (2016) & LF at $2.2<z<3.01500 \AA$ A rest frame \\
02 & -20.20 & 0.00532 & -1.31 & Parsa et al. (2016) & LF at $z=2.81500 \AA$ rest frame \\
03 & -20.71 & 0.00206 & -1.43 & Parsa et al. (2016) & LF at $z=3.81500 \AA$ rest frame \\
04 & -20.61 & 0.00161 & -1.54 & Parsa et al. (2016) & best fit at $z=3.31500 \AA$ rest frame \\
05 & -20.45 & 0.00410 & -1.36 & Weisz et al. (2014) & LF $z=3.01500 \AA$ rest frame \\
06 & -21.40 & 0.00086 & -1.50 & Cucciati et al. (2012) & $1500 \AA$ rest frame \\
07 & -20.94 & 0.00179 & -1.65 & van der Burg et al. (2010) & $z=3.01600 \AA$ rest frame \\
08 & -20.97 & 0.00171 & -1.73 & Reddy \& Steidel (2009) & $z=3.051700 \AA$ rest frame \\
09 & -20.90 & 0.00167 & -1.43 & Sawicki \& Thompson (2006) & $z=3.01700 \AA$ rest frame \\
\hline
\end{tabular}

Notes. Cucciati et al. (2012) did not actually measure the faint end slope at $z>1.7$, but fixed this value based on luminosity functions at lower redshifts.

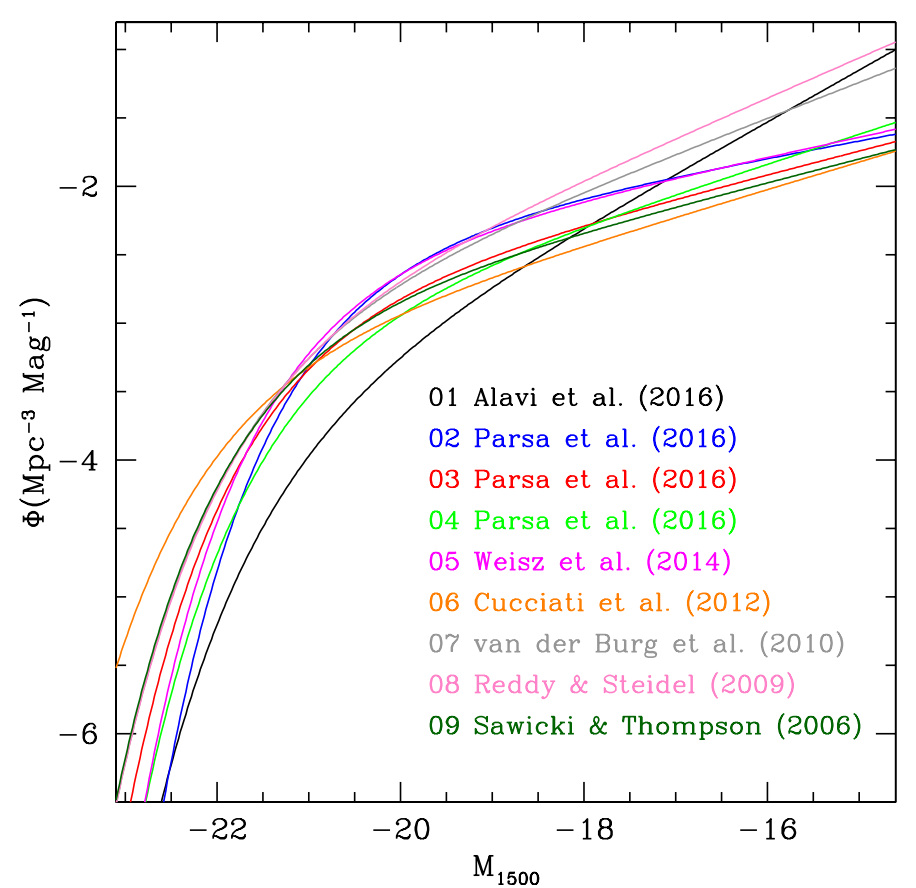

Fig. 6. Galaxy luminosity functions at $z \sim 3$ from different work, as summarized in Table 3.

We derive the relevant quantities needed to compute $\Gamma_{-12}$ in the following sections.

\subsection{Luminosity function of galaxies at $z \sim 3$}

An accurate estimate of the shape of the luminosity function at the faint end is crucial in order to quantify the luminosity density $\rho_{1500}$ and hence the H I photoionization rate produced by SFGs at $z \sim 3$. As in Grazian et al. (2016), we explored different determinations for the luminosity function of star-forming galaxies at these redshifts. We collected relevant luminosity functions at $z \sim 3$ from the literature, exploring the last ten years. In Table 3 we summarize the adopted parameters for the Schechter function $\left(M^{*}, \Phi^{*}, \alpha\right)$ of these recent works. Figure 6 shows the different shapes of the galaxy luminosity functions at $z \sim 3$.

A recent collection of luminosity functions from the literature has been provided by Parsa et al. (2016). They compute the luminosity function in two redshift bins, $z=2.8$ and $z=3.8$.
Moreover, they also provide an analytic formula to derive the parameters of the Schechter function depending on redshift. In Table 3 we consider the best fit of the relations given by Parsa et al. (2016) at $z=3.3$. The steep luminosity functions by van der van der Burg et al. (2010) and Reddy \& Steidel (2009) are not in agreement with the flatter $(\alpha \sim-1.4)$ functions at $z \sim 3$ (Parsa et al. 2016). A notable exception is the result of Alavi et al. (2016), with a slope of -1.9 and extending down to $M_{1500}=-12.5$, thanks to strong lensing magnification. It is interesting to note that the luminosity functions derived on lensing fields are usually steeper than those obtained on blank fields (see also Livermore et al. 2017; and Bouwens et al. 2016b, for similar comparisons at $z \sim 6$ ). This could be related to possible still unknown biases linked to the lensing amplification or completeness corrections. However, the main aim here is not to do a critical review of all the luminosity functions in the literature, but to select a representative sample in order to explore the implications on the calculations of the photoionizing background.

In Fig. 6 we note a large variance in the galaxy number density, especially at faint luminosities. At luminosities brighter than $0.5 L^{*}\left(M_{1500} \leq-20.2\right)$ this scatter does not influence the estimate of the photoionization rate $\Gamma_{-12}$; as already shown by Grazian et al. (2016), the contribution of bright galaxies is not relevant, irrespective of the adopted parameterization of the luminosity function. This is mainly due to the relatively low escape fraction $(\$ 2 \%)$ and to the low number densities at these bright luminosities. At fainter magnitudes, we can identify in Fig. 6 three main families of luminosity functions: 1) high space densities both at intermediate and faint magnitudes, with $\Phi \sim$ $5 \times 10^{-3} \mathrm{Mpc}^{-3} \mathrm{Mag}^{-1}$ at $M_{1500}=-19$ and $\Phi \sim 4 \times 10^{-2}$ at $M_{1500}=-16$ (07-van der Burg et al. 2010, 08-Reddy \& Steidel 2009); 2) high space density at intermediate magnitudes but low density at fainter magnitudes, with $\Phi \sim 5 \times 10^{-3}$ at $M_{1500}=-19$ and $\Phi \sim 10^{-2}$ at $M_{1500}=-16(02$-Parsa et al. $2016 z=2.8,05-$ Weisz et al. 2014); and 3) low space densities both at intermediate and faint magnitudes, with $\Phi \sim 2 \times 10^{-3}$ at $M_{1500}=-19$ and $\Phi \sim 10^{-2}$ at $M_{1500}=-16$ (03-Parsa et al. 2016, $z=$ 3.8; 04-Parsa et al. 2016 best fit at $z=3.3$, 06-Cucciati et al. 2012, 09-Sawicki \& Thompson 2006). The luminosity function of Alavi et al. (2016) is an exception in these three groups, with very low space density around $L^{*}$, but a steep faint end $(\alpha \sim-1.9)$.

In the next section we use these luminosity functions to evaluate the photoionization rate for different evolving escape fractions with luminosities $f_{\mathrm{esc}}^{\mathrm{rel}}(L)$. 
Table 4. H I photoionization rate $\Gamma_{-12}$ produced by galaxies for different functional forms of $f_{\mathrm{esc}}^{\text {rel }}(L)$ and for different parameterizations of the luminosity functions.

\begin{tabular}{c|ccccc|cccccc}
\hline \hline Models & $\mathrm{A}$ & $\mathrm{B}$ & $\mathrm{C}$ & $\mathrm{D}$ & $\mathrm{E}$ & \multicolumn{2}{|c|}{$\mathrm{A}$} & $\mathrm{B}$ & $\mathrm{C}$ & $\mathrm{D}$ & $\mathrm{E}$ \\
\hline $\mathrm{LF}$ & \multicolumn{4}{|c}{$\Gamma_{-12}(-26.0$} & $\left.\leq M_{1500} \leq-17.4\right)$ & \multicolumn{3}{c}{$\Gamma_{-12}\left(-26.0 \leq M_{1500} \leq-16.0\right)$} \\
\hline 01 & 0.04 & 0.22 & 0.39 & 0.34 & 0.19 & 0.06 & 0.34 & 0.65 & 0.62 & 0.56 \\
02 & 0.12 & 0.54 & 0.86 & 0.55 & 0.27 & 0.14 & 0.64 & 1.08 & 0.76 & 0.47 \\
03 & 0.09 & 0.35 & 0.55 & 0.49 & 0.26 & 0.10 & 0.42 & 0.71 & 0.65 & 0.47 \\
04 & 0.07 & 0.30 & 0.49 & 0.41 & 0.21 & 0.08 & 0.37 & 0.66 & 0.58 & 0.42 \\
05 & 0.13 & 0.53 & 0.83 & 0.63 & 0.32 & 0.15 & 0.63 & 1.05 & 0.85 & 0.56 \\
06 & 0.08 & 0.27 & 0.41 & 0.53 & 0.32 & 0.09 & 0.32 & 0.53 & 0.65 & 0.58 \\
07 & 0.13 & 0.52 & 0.86 & 0.86 & 0.48 & 0.15 & 0.68 & 1.20 & 1.21 & 1.04 \\
08 & 0.14 & 0.59 & 0.99 & 1.00 & 0.57 & 0.17 & 0.80 & 1.45 & 1.48 & 1.33 \\
09 & 0.09 & 0.33 & 0.50 & 0.50 & 0.27 & 0.10 & 0.39 & 0.64 & 0.64 & 0.48 \\
\hline Average & 0.10 & 0.41 & 0.65 & 0.59 & 0.32 & 0.12 & 0.51 & 0.89 & 0.83 & 0.66 \\
\hline
\end{tabular}

\subsection{Estimating the HI photoionization rate}

Table 4 summarizes the resulting photoionization rate $\Gamma_{-12}$ for different parameterizations of $f_{\mathrm{esc}}^{\mathrm{rel}}(L)$ and for different luminosity functions (Models A-E and luminosity functions 01-09, respectively). The relevant emissivities were computed integrating the luminosity function from $M_{1500}=-26.0$ to $M_{1500}=-17.4$ mag, corresponding to luminosities $4 \times 10^{-2} L^{*} \leq L \leq 10^{2} L^{*}$. The lower bound in luminosity was set to the faintest lensed galaxy for which we have an upper limit to the LyC escape fraction, the one determined by Amorin et al. (2014). We also provide in Table 4 the $\Gamma_{-12}$ obtained extending the lower limit to $M_{1500}=-16.0 \mathrm{mag}$, corresponding to a luminosity $L=$ $10^{-2} L^{*}$. For comparison, a recent estimate of the H I photoionization rate of $\Gamma_{-12}=0.79_{-0.19}^{+0.28}$ at $z=3.2$ has been provided by Becker \& Bolton (2013, hereafter BB13). We should take into account the contribution of the AGN population to $\Gamma_{-12}$ : a conservative estimate by Haardt \& Madau (2012) indicates $\Gamma_{-12}=0.37$ at $z \sim 3$ (see their Fig. 8a). Similarly, Cristiani et al. (2016) find a similar value $\Gamma_{-12}=0.36$ at $z=3.3$. Thus, a contribution by galaxies of at least $\Gamma_{-12} \sim 0.4$ is required in order to explain the measured UVB.

In order to interpret Table 4, we can divide the Models A-E into three categories: the first includes Model A (constant escape fraction of $1.7 \%$ at all luminosities); the second group (Models B and C) is characterized by low $f_{\mathrm{esc}}^{\text {rel }} \sim 1-2 \%$ for galaxies brighter than $M_{1500} \sim-20$ and $f_{\text {esc }}^{\text {rel }} \sim 10-20 \%$ for fainter objects; and finally, a third group (Models D and E) has $f_{\mathrm{esc}}^{\text {rel }} \gtrsim 20 \%$ fainter than $M_{1500} \sim-18$, and small values $f_{\text {esc }}^{\text {rel }} \leq 10 \%$ brighter than $M_{1500} \sim-19$. As mentioned above, the luminosity functions can also be divided into three main families according to the space densities at $M_{1500} \sim-19$ and $M_{1500} \sim-16$.

Looking at the left side of Table 4 (i.e., considering only galaxies brighter than $M_{1500}=-17.4$ ), it is easy to conclude that Model A has $\Gamma_{-12} \sim 0.04-0.14$, much lower than the required UVB value of 0.4 , for all the luminosity functions. Assuming a two-step function for $f_{\mathrm{esc}}^{\mathrm{rel}}(L)$ (Model B), only the luminosity functions with high space density at $M_{1500} \sim-19$ can provide enough $\mathrm{HI}$ ionizing photons at $z \sim 3$. Models $\mathrm{C}$ and $\mathrm{D}$ are instead able to reach the required UVB for all the luminosity functions taken into account. If the $\mathrm{LyC}$ escape fraction increases exponentially at lower luminosities (Model E), then only the luminosity functions of van der Burg et al. $(2010, \mathrm{LF}=07)$ and Reddy \& Steidel (2009, LF = 08), with high space densities both at $M_{1500} \sim-19$ and $M_{1500} \sim-16$, are able to reach the critical value for $\Gamma_{-12}$.
It is interesting to note that Model $\mathrm{C}$ provides a greater contribution to $\Gamma_{-12}$ than the other models considered. Under this scenario, galaxies at $M_{1500} \sim-19$ could be the main contributors to the ionizing UVB and are crucial for our understanding of the role of star formation in the reionization and post-reionization phases.

The results above are valid if we take into account galaxies brighter than $M_{1500}=-17.4$, or equivalently $L=0.036 L^{*}$. If we extend the lower bound to $M_{1500}=-16.0$ (right side of Table 4) or even fainter, we obtain larger values of $\Gamma_{-12}$. In particular, all the considered luminosity functions are always able to reach the required UVB, except for constant escape fractions at the level of $\sim 1-2 \%$ (Model A). Models with rapid increase of $f_{\mathrm{esc}}^{\text {rel }}$ at faint luminosities (Models B, C, D, and E) give $\Gamma_{-12}$ consistent with BB13, or even a factor of 2-3 higher when they are integrated down to $M_{1500}=-13.5$. In this case, extremely faint galaxies with luminosities between $10^{-3} L^{*}$ and $10^{-2} L^{*}$ are important in order to reproduce the UVB at $z \sim 3$, provided that the escape fraction is larger than $2 \%$ for all the faint star-forming galaxies. These regimes of luminosities will hopefully be investigated in the future thanks to strong lensing magnification (e.g., Vanzella et al. 2012b).

\section{Discussion}

\subsection{Comparison with predictions by theoretical models}

It is interesting at this stage to compare the observed limits for $f_{\text {esc }}^{\text {rel }}$ shown in Fig. 5 with predictions from theoretical models. In general, many theoretical models predict increasing ionizing escape fractions with progressively fainter galaxies. A recent exception to this scenario is provided by the Sharma et al. (2017) model where the escape fraction increases with the star formation surface density $\Sigma_{\mathrm{SF}}$ on scales of $1 \mathrm{kpc}$ reaching $5 \%$ and $13 \%$ in galaxies with $M_{1500}=-21.4$ and -23.9 , respectively. These predicted values are in contrast with our limits, which have been observed in galaxies in the same luminosity interval. We note that the starburst galaxy cited by Sharma et al. (2017) as a clear prototypical example of luminous LyC emitter shows X-ray detection probably associated with AGN activity (see Guaita et al. 2016).

Price et al. 2016 and Faisst (2016) derived empirical constraints from low-redshift observations in order to get constraints on the escape fraction and LyC production efficiency of galaxies. They assume a redshift evolution of the absolute escape fraction starting at $z=3.3$ from values in the range $f_{\mathrm{esc}}^{\text {abs }}=3.5-9 \%$, in tension with our observed value derived for bright galaxies 
$\left(M_{1500} \leq-20.0\right)$. Adopting values of $1-2 \%$, in better agreement with our limits, would imply a rapid evolution of the escape fraction with a quick increase up to the maximum redshift $z=8-9$ allowed by the late reionization scenario suggested by the Planck Collaboration XLVII (2016) data. It is also interesting to note that the empirical relation between the $[\mathrm{OIII}] /[\mathrm{OII}]$ ratio and $f_{\text {esc }}^{\text {abs }}$ adopted by Faisst (2016, Fig. 2) is not consistent with the observations of Christensen et al. (2012), Amorin et al. (2014), Bayliss et al. (2014). At $z>3$ and at relatively faint magnitudes, indeed, the latter have found that $f_{\mathrm{esc}}^{\mathrm{rel}} \leq 1 \%$ and $23 \%$ at $M_{1500}=-20.5$ and $M_{1500}=-17.4$, respectively, while the observed line ratio is $[\mathrm{OIII}] /[\mathrm{OII}]>10$. It is thus important to note that a causal connection between the high ionization efficiency and the large escape fraction of ionizing radiation has not been established yet.

Kimm et al. (2017), Anderson et al. (2017), Gnedin (2016), Hassan et al. (2016), Xu et al. (2016), Yoshiura et al. (2016) have used numerical cosmological simulations and theoretical tools to analyze the contribution of faint galaxies to the reionization process. From these studies it appears that the contribution to the reionization by star-forming galaxies does not strongly depend on redshift. For this reason, their estimates can be compared to the lower average redshift $z \sim 3.3$ of our sample. The predicted escape fractions are in general constant or increasing for progressively fainter galaxies, with values reaching up to $40-60 \%$, but as low as a few percent for the brighter fraction $\left(M_{1500}<-20\right)$.

In summary, all the recent theoretical models, with the exception of Sharma et al. (2016) and Sharma et al. (2017), tend to agree on relatively small values for the escape fraction at $z \sim 3$ and at luminosities close to $L^{*}$. They are thus in agreement with the observational results summarized in Fig. 5.

\subsection{Comparison with recent works on LyC escape fraction of star-forming galaxies}

In this section we compare our results, summarized in Fig. 5 and Table 2, with recent measurements of the escape fraction of starforming galaxies at high $z$. We divided the literature papers in two main groups, the first containing results based on the indirect signatures of possible $\mathrm{LyC}$ emission, while the second one summarizes the evidences of direct LyC detections.

\subsubsection{Indirect LyC measurements with other proxies}

The long search for LyC emitters over the last 15 years has stimulated the investigation of indirect proxies to pinpoint robust samples of $\mathrm{HI}$ ionizers in the local Universe and at high redshift. An efficient method, based on high [OIII]/[OII] line ratios or other high excitation lines, has been proposed by a number of works (e.g., Nakajima \& Ouchi 2014; Stark et al. 2015; Schaerer et al. 2016) and has been supported by the results of Izotov et al. (2016a,b), and Vanzella et al. (2016).

Usually, the high ionization efficiency of LyC emitter candidates is also associated with compact morphology and possibly strong outflows. Recently, Nakajima et al. (2016), Trainor et al. (2016), and Faisst (2016) have proposed searching for LyC emitters in galaxies with high ionizing ratios that are possibly due to the low metallicity or to the harder ionization spectrum (e.g., by a top-heavy initial mass function). Reddy et al. (2016) and Leethochawalit et al. (2016), instead, suggested searching for LyC emitters among the sources with low covering fractions of neutral hydrogen where the ISM is porous and has holes (the ionization-bound nebula of Zackrisson et al. 2013).

While all these methods are promising, it is worth stressing, however, that they are far from being robust indicators of appreciable LyC emission. The two notable examples discussed above, the galaxies cited by Amorin et al. (2014) and by Vasei et al. (2016), showed high [OIII]/[OII] line ratios coupled with upper limits on LyC escape fraction at the 10-20\% level, obtained through direct measurements. Moreover, Rutkowski et al. (2016) find that strong H- $\alpha$ emitters at $z \sim 1$, which are thought to be close analogs of high redshift sources of reionization, show an escape fraction $\leq 9.6 \%$ (at $3 \sigma$ ), again through direct LyC measurements. This suggests the need for more accurate calibrations of the indirect methods with direct LyC measurements.

\subsubsection{Direct LyC measurements}

Micheva et al. (2017) presented a sample of 18 LAEs and 7 LBGs detected in LyC out of a sample of 159 LAEs and $136 \mathrm{LBG}$ in the SSA22 field. The relative escape fraction for the LyC detected LAEs is $\sim 30 \%$, while for the LyC detected LBGs it is $\sim 20 \%$. However, a large fraction of the LyC candidates shows spatial offsets between the rest-frame non-ionizing detection and the LyC-emitting substructure or between the Lyman- $\alpha$ emission and the $\mathrm{H} \mathrm{I}$ ionizing radiation casting doubts on the fraction of contamination by lower redshift interlopers.

Smith et al. (2016) have used the Early Release Science (ERS) data of the UV sensitive WFC3 instrument on board HST to measure the LyC escape fraction for a sample of 50 massive SFGs at $z \sim 2.3-5.8$. They reported a $>3 \sigma$ detection of $f_{\text {esc }}^{\text {abs }} \sim 0.1 \%$ at $z \sim 2.4-2.7$ and a marginal $\leq 3 \sigma$ LyC signal at $z \sim 3$. These data do not show any significant correlation of $f_{\mathrm{esc}}^{\text {abs }}$ with the galaxy luminosity.

A different approach has recently been adopted by Shapley et al. (2016). They started from deep Keck/LRIS UV spectroscopy for a large sample of LBGs at $z \sim 3$, as described by Reddy et al. (2016). Roughly $10 \%$ of their galaxies show detections of LyC radiation (Steidel et al., in prep.). In particular, Shapley et al. (2006) studied the case of an intriguing spectroscopic LyC detection at $z \sim 3.2$ corroborated by HST data, which allows them to exclude contamination by foreground objects. This galaxy has $f_{\text {esc }}^{\text {abs }} \sim 42 \%$, but also shows evidence of high column density of neutral hydrogen, since the Lyman- $\beta$ and Lyman- $\gamma$ lines in absorption are saturated. Moreover, the age of this galaxy $(1.3 \mathrm{Gyr})$ is not as young as expected for strong LyC emitters, which should have young stellar populations of several Myr to provide the required ionizing photon efficiency.

Recently, starting from the stack spectrum of 33 galaxies at $z \sim 4$ from the VUDS survey, Marchi et al. (2017) have derived $\mathrm{a} \sim 2 \sigma$ detection of LyC radiation, measuring $f_{\mathrm{esc}}^{\mathrm{rel}}=0.09 \pm 0.04$. They also identified a possible LyC emitting galaxy at $z \sim 3.6$ with high EW in Lyman- $\alpha$. These results are in agreement with previous results (Vanzella et al. 2010; Boutsia et al. 2011; Grazian et al. 2016; Guaita et al. 2016; Japelj et al. 2017) and with our findings that the contribution of the whole galaxy population to the ionizing background is probably modest, extending the study at $z \sim 4$.

In summary, the few detections of LyC emission from SFGs at $z \geq 3$ indicate that they are rare events or that the distribution of escape fraction is bimodal. Only a limited subsample (a few percent) of the distant galaxies presents significant ( 20-40\%) escape fraction, while the general galaxy population usually does 
not show large $f_{\text {esc }}$ of ionizing photons (but see Steidel et al., in prep.). Our result, where no individual robust detection has been found out of a sample of 69 SFGs at $z \sim 3.3$, is in agreement with the recent achievements outlined above by Smith et al. (2016), Vanzella et al. (2016), Shapley et al. (2006), Guaita et al. (2016), Micheva et al. (2017), Japelj et al. (2017), and Marchi et al. (2017). It is thus possible that the escape of ionizing radiation is a peculiar phenomenon, associated with particular phases of the galaxy lifetime or due to the action of supernovae/AGN feedback on their ISM.

\section{Summary and conclusions}

We have used ultra-deep observations $(\gtrsim 30 \mathrm{~h})$ of the CANDELS/GOODS-North field with the LBC instrument in the $U$ and $R$ bands with the aim of determining the LyC escape fraction for a sample of faint $\left(L<0.5 L^{*}\right)$ star-forming galaxies at $z \sim 3.3$. To this end, we complemented the CANDELS/GOODSNorth sample with additional galaxies from the EGS and COSMOS fields, reaching a total of 69 star-forming galaxies of $-23 \lesssim M_{1500} \lesssim-19$ in the redshift interval $3.27<z<3.40$. This paper is thus an extension of the work by Grazian et al. (2016), both on the statistics (almost doubling the sample) and on the luminosity range analyzed.

Several ingredients are needed in order to reliably compute the LyC escape fraction: 1) very deep images at 900 and $1500 \AA$ rest frame, in this case the $U$ and the $R$ bands at $z \sim 3$;2) images with high spatial resolution, possibly from HST in different filters, in order to clean the sample from lower redshift contaminants; 3) high quality spectroscopic redshifts in a particularly narrow redshift range, in this case $3.27<z<3.40$, allowing the U filter of LBC to sample only LyC photons close to $900 \AA$ restframe; 4) high number statistics in order to reduce the stochasticity of the IGM absorption; and 5) deep X-ray data in order to exclude the AGNs from our sample.

We chose the CANDELS/GOODS-North, EGS, and COSMOS datasets since in all the three fields we have these ingredients, i.e., deep LBC data in the $U$ and in the $R$ bands in wide areas; HST coverage from CANDELS, AEGIS, and COSMOS; X-ray images from Chandra; and spectroscopic redshifts from the CANDELS, AEGIS, and VUDS-COSMOS databases. In particular, the critical ingredient of this work is the ultra-deep $U$-band image of LBC in the CANDELS/GOODS-North field covering an area of $\sim 0.6$ sq. deg to a limit of $30.2 \mathrm{AB}$ mag (at $1 \sigma)$. This image allows us to put stringent limits to the LyC escape fraction of faint star-forming galaxies at $z \sim 3$.

We cleaned the $z \sim 3$ sample from the contamination of AGNs and lower redshift interlopers, thanks to the Chandra and HST data. We used an efficient technique to compute the escape fraction from deep LBC data, based on the PSF-matched photometry, as carried out in Grazian et al. (2016). This reduces the uncertainties on the photometry and thus on the leakage of ionizing photons.

In order to explore the very faint regime, we added to our sample a lensed galaxy studied by van der Wel et al. (2013) and Amorin et al. (2014). It is a star-forming galaxy in the COSMOS field at $z=3.4$ lensed by an elliptical at $z \sim 1.5$. The upper limit in the $U$ band by LBC allows us to place an upper limit to the escape fraction of $23 \%$ for an object with luminosity of $0.036 L^{*}$. We added two other lensed galaxies from the literature (Christensen et al. 2012; Bayliss et al. 2014). For the last two galaxies we do not have deep LBC images in the $U$ band, and the upper limit to the $\mathrm{LyC}$ escape fraction comes from the estimate of the column density of neutral hydrogen from deep spectra in the Lyman- $\alpha$ region. The lensed galaxies reported in Christensen et al. (2012), Amorin et al. (2014), and Bayliss et al. (2014) are particularly important since they are intrinsically faint (and low mass) objects with high $[\mathrm{OIII}] /[\mathrm{OII}]$ line ratios. They thus represent the interesting subclass of galaxies with high ionization efficiency that are supposed to emit a very large number of ionizing photons.

We derive an upper limit to the LyC $f_{\text {esc }}^{\text {rel }}$ for each individual galaxy of the sample. We also stack the $U$ and $R$ bands of our sample to derive a limit for the whole population of SFGs at $z \sim 3$. The main results are the following:

- Out of 69 galaxies, we detect LyC emission only in 3 (two discussed in Grazian et al. 2016, and the third in the EGS field, ID = 11372). The source ID = 16479 in the CANDELS/GOODS-North field was discarded because it is possibly affected by contamination.

- Stacking the $U$ - and the $R$-band images of the whole sample, we derive an upper limit of $f_{\mathrm{esc}}^{\mathrm{rel}} \leq 1.7 \%$ at $S / N=1$, more stringent than previous results (e.g., Chen et al. 2007; Vanzella et al. 2010; Boutsia et al. 2011; Grazian et al. 2016; Guaita et al. 2016; Japelj et al. 2017; Marchi et al. 2017).

- Dividing the total sample in different luminosity intervals, we derive limits of $f_{\mathrm{esc}}^{\mathrm{rel}} \leq 2.5 \%, 2.0 \%, 2.4 \%$, and $10.3 \%$ at $M_{1500}=-22.8, M_{1500}=-21.7, M_{1500}=-20.8$, and $M_{1500}=-19.7$, respectively. The apparent increase in the escape fraction toward faint luminosities is due to an observational limit in the depth of the $U$ band.

- We explored different dependencies of the relative escape fraction on the galaxy luminosity, and provided different parameterizations to reproduce the observed trends of $f_{\mathrm{esc}}^{\mathrm{rel}}(L)$.

- We combined the derived parametric forms of $f_{\mathrm{esc}}^{\mathrm{rel}}(L)$ with different realizations of the galaxy luminosity function at $z \sim$ 3 taken from the literature, and we evaluated the emissivity at $900 \AA$ rest-frame, $\rho_{900}^{\text {esc }}$.

- Translating this quantity into a neutral hydrogen photoionization rate $\Gamma_{-12}$, we can conclude that $z \sim 3$ galaxies brighter than $M_{1500}=-17.4$ have difficulties providing the UVB measured in the Lyman forest at $z \sim 3$, especially if their LyC escape fraction is below $10 \%$.

- Fainter galaxies $\left(M_{1500} \leq-16\right)$ can produce the observed UVB if their LyC escape fraction is above $10 \%$ at low luminosities. If a fainter limit of $M_{1500} \sim-13$ is adopted, then the constraints on $f_{\mathrm{esc}}^{\text {rel }}$ can be relaxed.

- For two lensed galaxies with extreme line ratios of $[\mathrm{OIII}] /[\mathrm{OII}]>10$ there are stringent upper limits of $f_{\mathrm{esc}}^{\mathrm{rel}} \leq 1 \%$ and $23 \%$ at $M_{1500}=-20.5$ and $M_{1500}=-17.4$, respectively (Bayliss et al. 2014; Amorin et al. 2014). Any causal connection between the high ionization efficiency and the large escape fraction of LyC radiation still awaits confirmation from a larger sample.

Placing these results in a cosmological context, it is interesting to note that if such low values of escape fraction are also found at $z>4$ and for faint luminosities, then star-forming galaxies would be insufficient to cause the $\mathrm{H}$ I reionization process.

Future work will include the derivation of a robust estimate of the faint end of the luminosity function at $z \sim 3$ in large areas thanks to the deep $U$-band images in the COSMOS (Boutsia et al. 2014), EGS, and ultra-deep CANDELS/GOODSNorth data (Grazian, et al., in prep.). The combination of wide and deep LBC data, together with large spectroscopic databases available in these fields, will allow us to measure the slope $\alpha$ with unprecedented precision. This measurement, as well as the 
measurements of the escape fraction, is a crucial item in the derivation of precise contribution of faint galaxies to the UVB at $z=3$. The CANDELS/GOODS-North sample can also be used in the future to confirm the spectroscopic redshifts for other star-forming galaxies at $3.27<z<3.40$ in the faint regime $(25.5<R<26.0)$ with deep optical spectroscopy. At present, in fact, in this work we use only 8 galaxies with spectroscopic redshifts available out of 48 galaxies (16\%) at $3.27 \leq z_{\text {phot }} \leq 3.40$ and $R \leq 26.0$ in this field. While this spectroscopic subsample is relatively small, it is nonetheless representative of the faint starforming galaxy population at $z \sim 3$, indicating that our results are not biased by low number statistics even at these faint limits. The spectroscopic completeness of galaxies at $R \leq 26.0$ can be enhanced in the future by adding $\sim 40$ galaxies and improving the limit of $f_{\mathrm{esc}}^{\text {rel }}$ down to $\sim 4 \%$.

In the future, it will be crucial to measure the escape fraction at faint magnitudes, $M_{1500} \sim-16$ for a large number of galaxies. With this aim, strong lensing magnification (either through galaxy-galaxy lensing or by massive clusters) will be a crucial instrument for exploring luminosity regimes that would otherwise be inaccessible with the present instrumentation. The rapid activities in this field (e.g., SLACS, SL2S surveys for galaxygalaxy lensing; HST Frontier Fields for lensing by clusters) will give fresh data in the near future.

Acknowledgements. We warmly thank the anonymous referee for the useful suggestions and constructive comments that helped us to improve this paper. We acknowledge the financial contribution from the agreement ASI-INAF I/009/10/0. The LBT is an international collaboration among institutions in the United States, Italy, and Germany. LBT Corporation partners are The University of Arizona on behalf of the Arizona university system; Istituto Nazionale di Astrofisica Italy; LBT Beteiligungsgesellschaft, Germany, representing the Max-Planck Society, the Astrophysical Institute Potsdam, and Heidelberg University; The Ohio State University; and The Research Corporation, on behalf of The University of Notre Dame, University of Minnesota, and University of Virginia. Based on observations collected at the European Organisation for Astronomical Research in the Southern Hemisphere under ESO programme(s) 185.A-0791. Based on observations obtained with MegaPrime/MegaCam, a joint project of CFHT and CEA/IRFU, at the Canada-France-Hawaii Telescope (CFHT) which is operated by the National Research Council (NRC) of Canada, the Institut National des Science de l'Univers of the Centre National de la Recherche Scientifique (CNRS) of France, and the University of Hawaii. This work is based in part on data products produced at Terapix available at the Canadian Astronomy Data Centre as part of the Canada-France-Hawaii Telescope Legacy Survey, a collaborative project of NRC and CNRS.

\section{References}

Adelberger, K. L., Steidel, C. C., Shapley, A. E., et al. 2004, ApJ, 607, 226 Alavi, A., Siana, B., Richard, J., et al. 2016, ApJ, 832, 56 Alexander, D. M., Bauer, F. E., Brandt, W. N., et al. 2003, AJ, 126, 539 Amorín, R., Perez-Montero, E., \& Vilchez, J. M. 2010, ApJ, 715, 128 Amorín, R., Perez-Montero, E., Vilchez, J. M., \& Papaderos, P. 2012a, ApJ, 749, 185

Amorín, R., Vilchez, J. M., Hagele, G. F., et al. 2012b, ApJ, 754, 22 Amorín, R., Grazian, A., Castellano, M., et al. 2014, ApJ, 788, L4 Amorín, R., Fontana, A., Perez-Montero, E., et al. 2017, Nat. Astron., 1, 0052 Anderson, L., Governato, F., Karcher, M., Quinn, T., \& Wadsley, J. 2017, MNRAS, 468, 4077

Ashcraft, T. A., Windhorst, R. A., Jansen, R. A., et al. 2017, PASP, submitted, [arXiv: 1703.09874]

Barger, A. J., Cowie, L. L., \& Wang, W.-H. 2008, ApJ, 689, 687

Bayliss, M. B., Rigby, J. R., Sharon, K., et al. 2014, ApJ, 790, 144

Becker, G. D., \& Bolton, J. S. 2013, MNRAS, 436, 1023

Becker, G. D., Bolton, J. S., Madau, P., et al. 2015, MNRAS, 447, 3402

Bergvall, N., Leitet, E., Zackrisson, E., \& Marquart, T. 2013, A\&A, 554, A38

Bertin, E., \& Arnouts, S. 1996, A\&AS, 117, 393

Borthakur, S., Heckman, T. M., Leitherer, C., \& Overzier, R. A. 2014, Science, 346, 216

Boutsia, K., Grazian, A., Giallongo, E., et al. 2011, ApJ, 736, 41

Boutsia, K., Grazian, A., Giallongo, E., et al. 2014, A\&A, 563, A142
Bouwens, R. J., Illingworth, G. D., Oesch, P. A., et al. 2015, ApJ, 811, 140 Bouwens, R. J., Smit, R., Labbé, I., et al. 2016a, ApJ, 831, 176 Bouwens, R. J., Oesch, P. A., Illingworth, G. D., et al. 2016b, ApJ, submitted, [arXiv: 1610.00283]

Bridge, C. R., Teplitz, H. I., Siana, B., et al. 2010, ApJ, 720, 465

Cardamone, C., Schawinski, K., Sarzi, M., et al. 2009, MNRAS, 399, 1191

Castellano, M., Dayal, P., Pentericci, L., et al. 2016, ApJ, 818, L3

Chardin, J., Puchwein, E., \& Haehnelt, M. G. 2017, MNRAS, 465, 3429

Chen, H.-W., Prochaska, J. X., \& Gnedin, N. Y. 2007, ApJ, 667, L125

Christensen, L., Laursen, P., Richard, J., et al. 2012, MNRAS, 427, 1973

Civano, F., Marchesi, S., Comastri, A., et al. 2016, ApJ, 819, 62

Cooper, M. C., Aird, J. A., Coil, A. L., et al. 2011, ApJS, 193, 14

Cowie, L. L., Barger, A. J., \& Trouille, L. 2009, ApJ, 692, 1476

Cristiani, S., Serrano, L. M., Fontanot, F., Vanzella, E., \& Monaco, P. 2016, MNRAS, 462, 2478

Cucciati, O., Tresse, L., Ilbert, O., et al. 2012, A\&A, 539, A31

Davis, M., Guhathakurta, P., Konidaris, N. P., et al. 2007, ApJ, 660, L1

de Barros, S., Vanzella, E., Amorín, R., et al. 2016, A\&A, 585, A51

De Santis, C., Grazian, A., Fontana, A., \& Santini, P. 2007, New Astron., 12, 271

Dijkstra, M., Gronke, M., \& Venkatesan, A. 2016, ApJ, 828, 71

Dunlop, J. S., Rogers, A. B., McLure, R. J., et al. 2013, MNRAS, 432, 3520

Ellis, R. S. 2014, Proceedings of the 26th Solvay Conference on Physics:

Astrophysics and Cosmology, eds. R. Blandford, \& A. Sevrin (World Scientific)

Eldridge, J. J., \& Stanway, E. R. 2009, MNRAS, 400, 1019

Faisst, A. L. 2016, ApJ, 829, 99

Fan, X., Strauss, M. A., Becker, R. H., et al. 2006, AJ, 132, 117

Faucher-Giguère, C. A., Lidz, A., Zaldarriaga, M., \& Hernquist, L. 2009, ApJ, 703, 1416

Ferrara, A., \& Loeb, A. 2013, MNRAS, 431, 2826

Finkelstein, S. L., Ryan, R. E. Jr., Papovich, C., et al. 2015, ApJ, 810, 71

Fontana, A., Vanzella, E., Pentericci, L., et al. 2010, ApJ, 725, L205

Franx, M., Labbé, I., Rudnick, G., et al. 2003, ApJ, 587, L79

Giallongo, E., Cristiani, S., D’Odorico, S., \& Fontana, A. 2002, ApJ, 568, L9

Giallongo, E., Grazian, A., Fiore, F., et al. 2015, A\&A, 578, A83

Giavalisco, M., Ferguson, H. C., Koekemoer, A. M., et al. 2004, ApJ, 600, L93

Glikman, E., Djorgovski, S. G., Stern, D., et al. 2011, ApJ, 728, L26

Gnedin, N. Y. 2016, ApJ, 825, L17

Gnedin, N. Y., Kravtsov, A. V., \& Chen, H.-W. 2008, ApJ, 672, 765

Grazian, A., Giallongo, E., Gerbasi, R., et al. 2016, A\&A, 585, A48

Grimes, J. P., Heckman, T., Aloisi, A., et al. 2009, ApJS, 181, 272

Grogin, N. A., Kocevski, D. D., Faber, S. M., et al. 2011, ApJS, 197, 35

Guaita, L., Pentericci, L., Grazian, A., et al., 2016, A\&A, 587, A133

Haardt, F., \& Madau, P. 1996, ApJ, 461, 20

Hassan, S., Davé, R., Finlator, K., \& Santos, M. G. 2016, MNRAS, 457, 1550

Heckman, T. M., Borthakur, S., Overzier, R., et al. 2011, ApJ, 730, 5

Henry, A., Scarlata, C., Martin, C. L., \& Erb, D. 2015, ApJ, 809, 19

Hinshaw, G., Larson, D., Komatsu, E., et. al. 2013, ApJS, 208, 19

Inoue, A. K., Shimizu, I., Iwata, I., \& Tanaka, M. 2014, MNRAS, 442, 1805

Izotov, Y. I., Orlitová, I., Schaerer, D., et al. 2016a, Nature, 529, 178

Izotov, Y. I., Schaerer, D., Thuan, T. X., et al. 2016b, MNRAS, 461, 3683

Japelj, J., Vanzella, E., Fontanot, F., et al. 2017, MNRAS, 468, 389

Khaire, V., Srianand, R., Choudhury, T. R., \& Gaikwad, P. 2016, MNRAS, 457, 4051

Khostovan, A. A., Sobral, D., Mobasher, B., et al. 2016, MNRAS, 463, 2363

Kimm, T., Katz, H., Haehnelt, M., et al. 2017, MNRAS, 466, 4826

Koekemoer, A. M., Faber, S. M., Ferguson, H. C., et al. 2011, ApJS, 197, 36

Konno, A., Ouchi, M., Ono, Y., et al. 2014, ApJ, 797, 16

Leethochawalit, N., Jones, T. A., Ellis, R. S., et al. 2016, ApJ, 831, 152

Le Fèvre, O., Tasca, L. A. M., Cassata, P., the VUDS Team 2015, A\&A, 576, A79

Leitet, E., Bergvall, N., Hayes, M., Linné, S., \& Zackrisson, E. 2013, A\&A, 553, A106

Leitherer, C., Hernandez, S., Lee, J. C., Oey, M. S. 2016, ApJ, 823, 64

Livermore, R. C., Finkelstein, S. L., \& Lotz, J. M. 2017, ApJ, 835, 113

Ma, X., Kasen, D., Hopkins, P. H., et al. 2015, MNRAS, 453, 960

Madau, P., \& Haardt, F. 2015 ApJ, 813, L8

Marchi, F., Pentericci, L., Guaita, L., et al. 2017, A\&A, 601, A73

Matthee, J., Sobral, D., Santos, S., et al. 2015, MNRAS, 451, 400

Micheva, G., Iwata, I., Inoue, A. K., et al. 2017, MNRAS, 465, 316

Mostardi, R. E., Shapley, A. E., Nestor, D. B., et al. 2013, ApJ, 779, 65

Naidu, R. P., Oesch, P. A., Reddy, N., et al. 2016, ApJ, submitted [arXiv: 1611.07038]

Nakajima, K., \& Ouchi, M. 2014, MNRAS, 442, 900

Nakajima, K., Ellis, R. S., Iwata, I., et al. 2016, ApJ, 831, L9

Nandra, K., Laird, E. S., Aird, J. A., et al. 2015, ApJS, 220, 10

Nestor, D. B., Shapley, A. E., Steidel, C. C., \& Siana, B. 2011, ApJ, 736, 18

Oesch, P. A., van Dokkum, P. G., Illingworth, G. D., et al. 2015, ApJ, 804, L30 
Ono, Y., Ouchi, M., Mobasher, B., et al. 2012, ApJ, 744, 83 Onodera, M., Carollo, C. M., Lilly, S., et al. 2016, ApJ, 822, 42

Parsa, S., Dunlop, J. S., McLure, R. J., \& Mortlock, A. 2016, MNRAS, 456, 3194

Pentericci, L., Fontana, A., Vanzella, E., et al. 2011, ApJ, 743, 132

Pentericci, L., Vanzella, E., Fontana, A., et al. 2014, ApJ, 793, 113

Pirzkal, N., Rothberg, B., Ly, C., et al. 2013, ApJ, 772, 48

Planck Collaboration XLVII. 2016, A\&A, 596, A108

Razoumov, A. O., \& Sommer-Larsen, J. 2010, ApJ, 710, 1239

Reddy, N. A., \& Steidel, C. C. 2009, ApJ, 692, 778

Reddy, N. A., Steidel, C. C., Erb, D. K., et al. 2006, ApJ, 653, 1004

Reddy, N. A., Steidel, C. C., Pettini, M., et al. 2016, ApJ, 828, 108

Rigopoulou, D., Huang, J.-S., Papovich, C., et al. 2006, ApJ, 648, 81

Robertson, B. E., Furlanetto, S. R., Schneider, E., et al. 2013, ApJ, 768, 71

Rutkowski, M. J., Scarlata, C., Haardt, F., et al. 2016, ApJ, 819, 81

Santos, S., Sobral, D., \& Matthee, J. 2016, MNRAS, 463, 1678

Sawicki, M., \& Thompson, D. 2006, ApJ, 642, 653

Schaerer, D., Izotov, Y. I., Verhamme, A., et al. 2016, A\&A, 591L, 8

Schenker, M. A., Ellis, R. S., Konidaris, N. P., \& Stark, D. P. 2014, ApJ, 795, 20

Schmidt, K. B., Treu, T., Bradac, M., et al. 2016, ApJ, 818, 38

Scoville, N., Aussel, H., Brusa, M., et al. 2007, ApJS, 172, 1

Siana, B., Teplitz, H. I., Colbert, J., et al. 2007, ApJ, 668, 62

Siana, B., Teplitz, H. I., Ferguson, H. C., et al. 2010, ApJ, 723, 241

Shapley, A. E., Steidel, C. C., Pettini, M., Adelberger, K. L., \& Erb, D. K. 2006, ApJ, 651, 688

Shapley, A. E., Steidel, C. C., Strom, A. L., et al. 2016, ApJ, 826, L24

Sharma, M., Theuns, T., Frenk, C., et al. 2016, MNRAS, 458, L94

Sharma, M., Theuns, T., Frenk, C., et al. 2017, MNRAS, 468, 2176

Smith, B., Windhorst, R. A., Jansen, R. A., et al. 2016, ApJ, submitted, [arXiv: 1602.01555]

Stanway, E. R., Eldridge, J. J., Becker, G. D., et al. 2016, MNRAS, 456, 485

Stark, D. P., Walth, G., Charlot, S., et al. 2015, MNRAS, 454, 1393

Stark, D. P., Ellis, R. S., Charlot, S., et al. 2017, MNRAS, 464, 469

Stasinska, G., Izotov, Yu., Morisset, C., \& Guseva, N. 2015, A\&A, 576, A83

Stefanon, M., Yan, H., Mobasher, B., et al. 2017, ApJS, 229, 32

Steidel, C. C., Pettini, M., \& Adelberger, K. L. 2001, ApJ, 546, 665

Steidel, C. C., Adelberger, K. L., Shapley, A. E., et al. 2003, ApJ, 592, 728

Steidel, C. C., Strom, A. L., Pettini, M., et al. 2016, ApJ, 826, 159

Trainor, R. F., Strom, A. L., Steidel, C. C., \& Rudie, G. C. 2016, ApJ, 832, 171

Treu, T., Trenti, M., Stiavelli, M., et al. 2012, ApJ, 747, 27

van der Burg, R. F. J., Hildebrandt, H., \& Erben, T. 2010, A\&A, 523, A74

van der Wel, A., van de Ven, G., Maseda, M., et al. 2013, ApJ, 777, L17

Vanzella, E., Giavalisco, M., Inoue, A. K., et al. 2010, ApJ, 725, 1011

Vanzella, E., Guo, Y., Giavalisco, M., et al. 2012a, ApJ, 751, 70

Vanzella, E., Nonino, M., Cristiani, S., et al. 2012b, MNRAS, 424, L54

Vanzella, E., Fontana, A., Zitrin, A., et al. 2014, ApJ, 783, L12

Vanzella, E., de Barros, S., Vasei, K., et al. 2016, ApJ, 825, 41

Vasei, K., Siana, B., Shapley, A. E., et al. 2016, ApJ, 831, 38

Verhamme, A., Orlitova, I., Schaerer, D., et al. 2017, A\&A, 597, A13

Weisz, D. R., Johnson, B. D., \& Conroy, C. 2014, ApJ, 794, L3

Wirth, G. D., Trump, J. R., Barro, G., et al. 2015, AJ, 150, 153

Worseck, G., Prochaska, J. X., O'Meara, J. M., et al. 2014, MNRAS, 445, 1745

Xu, H., Wise, J. H., Norman, M. L., Ahn, K., \& O'Shea, B. W. 2016, ApJ, 833, 84

Yajima, H., Choi, J.-H., \& Nagamine, K. 2011, MNRAS, 412, 411
Yoshiura, S., Hasegawa, K., Ichiki, K., et al. 2016, MNRAS, submitted, [arXiv: 1602.04407]

Zahn, O., Reichardt, C. L., Shaw, L., et al. 2012, ApJ, 756, 65

1 INAF-Osservatorio Astronomico di Roma, via Frascati 33, 00078 Monte Porzio Catone, Italy e-mail: andrea.grazian@oa-roma.inaf.it

${ }^{2}$ Carnegie Observatories, Colina El Pino, Casilla 601 La Serena, Chile

3 National Optical Astronomy Observatory, 950 North Cherry Ave, Tucson, AZ 85719, USA

${ }^{4}$ School of Earth and Space Exploration, Arizona State University, Tempe, AZ 85287-1404, USA

5 Minnesota Institute for Astrophysics, University of Minnesota, 116 Church Street SE, Minneapolis, MN 55455, USA

6 Stockholm University, Alba Nova SCFAB, 10691 Stockholm, Sweden

7 INAF-Osservatorio Astronomico di Bologna, via Ranzani, 1, 40127 Bologna, Italy

8 INAF-Osservatorio Astronomico di Trieste, via G.B. Tiepolo, 11 34143 Trieste, Italy

9 Astronomy Department, University of Massachusetts, Amherst, MA 01003, USA

10 Space Telescope Science Institute, 3700 San Martin Drive, Baltimore, MD 21218, USA

11 Cavendish Laboratory, University of Cambridge, 19 J.J. Thomson Ave., Cambridge CB30HE, UK

12 Kavli Institute of Cosmology c/o Institute of Astronomy, Madingley Road, Cambridge CB3 0HA, UK

13 Department of Astronomy and Astrophysics, University of California Berkeley 501 Campbell Hall Berkeley, CA 94720, USA

14 Agenzia Spaziale Italiana Science Data Center, via del Politecnico snc, 00133 Roma, Italy

15 UCO/Lick Observatory, 1156 High Street Santa Cruz, CA 95064, USA

16 INAF-IASF, via Bassini 15, 20133 Milano, Italy

17 Max Planck Institute for extraterrestrial Physics, Giessenbachstrasse 185748 Garching, Bayern, Germany

18 ESO Vitacura, Alonso de Cordova 3107, Vitacura, Casilla 19001, Santiago de Chile, Chile

19 University of Kentucky, 600 Rose Street, Lexington, KY 40508, USA

20 Department of Astronomy, University of Virginia, Charlottesville, VA 22904-4325, USA

21 Department of Astronomy and Steward Observatory, University of Arizona, Tucson, AZ 85719, USA

22 Department of Physics and Astronomy University of Missouri Columbia, MO 65211, USA 